\title{
Genomes of early-diverging streptophyte algae shed light on plant terrestrialization
}

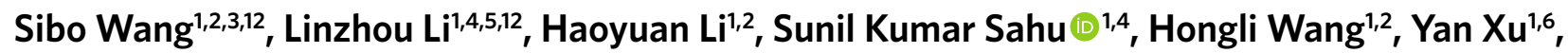 \\ Wenfei Xian ${ }^{1,2}$, Bo Song ${ }^{1,2}$, Hongping Liang ${ }^{1,6}$, Shifeng Cheng ${ }^{1,2}$, Yue Chang ${ }^{1,2}$, Yue Song ${ }^{1,2}$, \\ Zehra Çebi', Sebastian Wittek7, Tanja Reder7, Morten Peterson ${ }^{3}$, Huanming Yang ${ }^{1,2}$, Jian Wang ${ }^{1,2}$, \\ Barbara Melkonian ${ }^{7,11}$, Yves Van de Peer ${ }^{8,9}$, Xun Xu ${ }^{1,2}$, Gane Ka-Shu Wong ${ }^{1,10 \star}$, Michael Melkonian ${ }^{7,11 \star}$, \\ Huan Liu ${ }^{1,3,4 \star}$ and Xin Liu ${ }^{1,2,4 \star}$
}

\begin{abstract}
Mounting evidence suggests that terrestrialization of plants started in streptophyte green algae, favoured by their dual existence in freshwater and subaerial/terrestrial environments. Here, we present the genomes of Mesostigma viride and Chlorokybus atmophyticus, two sister taxa in the earliest-diverging clade of streptophyte algae dwelling in freshwater and subaerial/terrestrial environments, respectively. We provide evidence that the common ancestor of $M$. viride and $C$. atmophyticus (and thus of streptophytes) had already developed traits associated with a subaerial/terrestrial environment, such as embryophyte-type photorespiration, canonical plant phytochrome, several phytohormones and transcription factors involved in responses to environmental stresses, and evolution of cellulose synthase and cellulose synthase-like genes characteristic of embryophytes. Both genomes differed markedly in genome size and structure, and in gene family composition, revealing their dynamic nature, presumably in response to adaptations to their contrasting environments. The ancestor of $\boldsymbol{M}$. viride possibly lost several genomic traits associated with a subaerial/terrestrial environment following transition to a freshwater habitat.
\end{abstract}

T: ransition to a terrestrial environment, termed terrestrialization, is generally regarded as a pivotal event in the evolution and diversification of land plant flora ${ }^{1}$. Extant green plants (Viridiplantae) can be subdivided into two lineages, Chlorophyta (most of the green algae) and Streptophyta (embryophytes and their closest algal relatives, a grade collectively known as streptophyte algae ${ }^{2}$. There is now compelling evidence that adaptation to subaerial/terrestrial habitats is a feature of streptophyte algae, arising from their dual existence in freshwater and subaerial/terrestrial environments throughout their evolutionary history. Recent transcriptomic and genomic studies have shown that the molecular toolkit for life in a terrestrial environment was already present in streptophyte algae ${ }^{3}$. Homologues of genes once thought to be restricted to embryophytes are now being detected in streptophyte algae. Examples include those involved in symbiotic or pathogenic interactions with soil microbes ${ }^{4}$, phytohormone signalling ${ }^{5-8}$, desiccation/stress ${ }^{9}$, plastid/nucleus retrograde signalling ${ }^{10,11}$ and cell wall metabolism ${ }^{12}$. Importantly, many transcription factors (TFs) thought to be specific to embryophytes originated in streptophyte algae and also substantially expanded there ${ }^{13}$. These findings raise exciting questions about the functional role of embryophyte-like genes in streptophyte algae.

In phylogenomic analyses, the earliest-diverging streptophyte algae are represented by a clade comprising two monospecific genera, Mesostigma and Chlorokybus ${ }^{14-17}$. Both are structurally simple but differ in their cellular organization, life history and type of habitat $^{18}$. Mesostigma viride is a scale-covered flagellate with an eyespot that reproduces by binary division at the flagellate stage (Fig. 1b). Chlorokybus atmophyticus consists of sarcinoid cell packets where each cell has its own cell wall, occasionally producing biflagellate, scaly zoospores (Fig. 1b). Importantly, M. viride is found in the benthos of small, shallow ponds whereas C. atmophyticus is a subaerial/ terrestrial alga that occurs among bryophytes, on soil and on stones.

Previous analyses of two streptophyte algal genomes, Klebsormidium nitens $s^{6}$ and Chara braunii ${ }^{7}$, have not only revealed embryophyte-like genomic traits with gains and expansions of respective genes/gene families in both taxa, but also loss of genes involved in responses to abiotic stresses that prevail in a terrestrial environment, in the aquatic (freshwater) C. braunii ( $K$. nitens thrives in a subaerial/terrestrial environment). The draft genomes of $M$. viride and C. atmophyticus reported here allowed us to address two questions important for plant terrestrialization: (1) did the common ancestor of streptophytes already display embryophyte-like genomic traits that would be indicative for adaptation to a terrestrial environment; and (2) how do the genomes of $M$. viride and C. atmophyticus differ from each other in light of previous genome studies on two streptophyte algae that occur in contrasting environments (subaerial/terrestrial and aquatic) but belong to different streptophyte classes (Klebsormidiophyceae and Charophyceae)?

'BGI-Shenzhen, Shenzhen, China. ${ }^{2}$ China National GeneBank, BGI-Shenzhen, Shenzhen, China. ${ }^{3}$ Department of Biology, University of Copenhagen, Copenhagen, Denmark. ${ }^{4}$ State Key Laboratory of Agricultural Genomics, BGI-Shenzhen, Shenzhen, China. ${ }^{5}$ Department of Biotechnology and Biomedicine, Technical University of Denmark, Lyngby, Denmark. ${ }^{6}$ BGI Education Center, University of Chinese Academy of Sciences, Shenzhen, China. ${ }^{7}$ Botanical Institute, Cologne Biocenter, University of Cologne, Cologne, Germany. ${ }^{8}$ Department of Plant Biotechnology and Bioinformatics, Ghent University and VIB/UGent Center for Plant Systems Biology, Ghent, Belgium. ${ }^{9}$ Centre for Microbial Ecology and Genomics, Department of Biochemistry, Genetics and Microbiology, University of Pretoria, Pretoria, South Africa. ${ }^{10}$ Department of Biological Sciences and Department of Medicine, University of Alberta, Edmonton, Alberta, Canada. "1Present address: University of Duisburg-Essen, Campus Essen, Faculty of Biology, Essen, Germany. ${ }^{12}$ These authors contributed equally: Sibo Wang, Linzhou Li. *e-mail: gane@ualberta.ca; michael.melkonian@uni-koeln.de; liuhuan@genomics.cn; liuxin@genomics.cn 


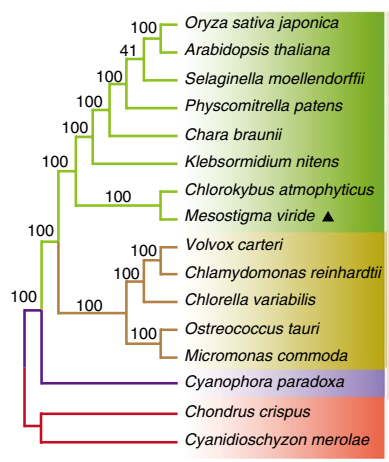

b

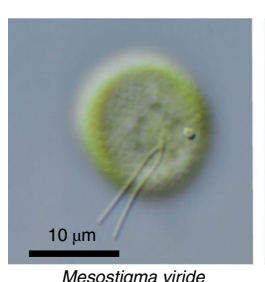

Mesostigma viride

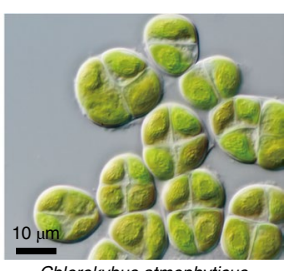

Chlorokybus atmophyticus

c
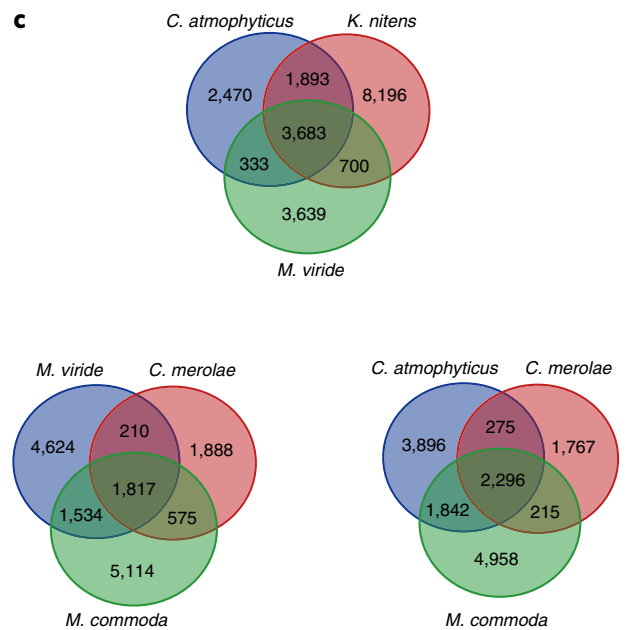

d

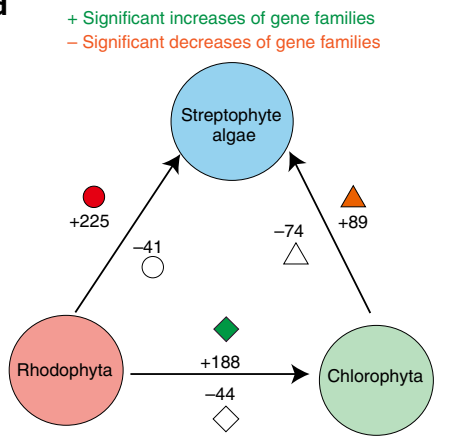

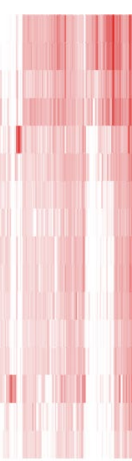
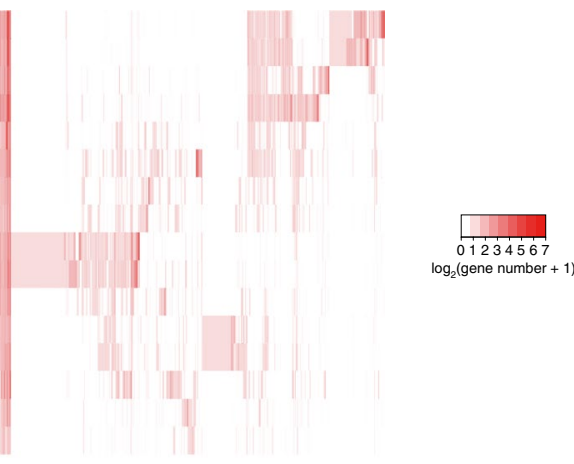

e

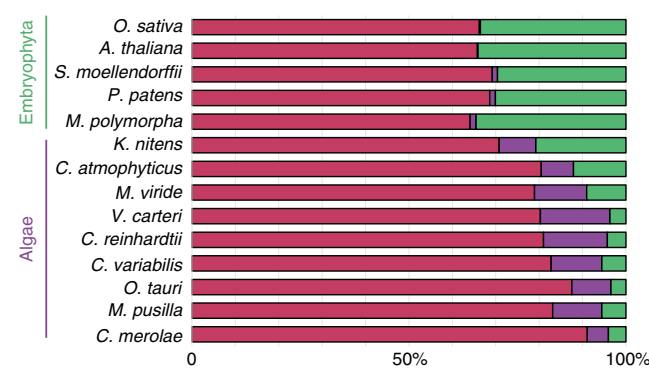

Algae and embryophyta

Alga

$\square$ Embryophyta

f

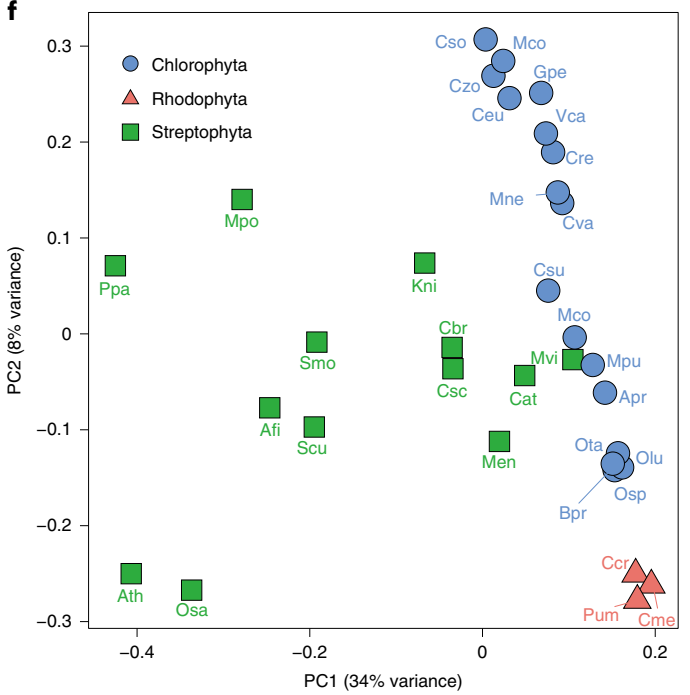

\begin{tabular}{|l|l|l|l|}
\hline \multicolumn{1}{|c|}{ Expansion } & & \multicolumn{1}{|c|}{ Contraction } \\
\hline \multirow{4}{*}{$\begin{array}{l}\text { Plant hormone signal transduction } \\
\text { Starch and sucrose metabolism } \\
\text { Photosynthesis:antenna proteins } \\
\text { Plant-pathogen interaction } \\
\text { Carbon fixation in photosynthetic organisms }\end{array}$} & $\bigcirc$ & $\begin{array}{l}\text { Alpha-linolenic acid metabolism } \\
\text { RNA polymerase } \\
\text { Pentose and glucuronate interconversions } \\
\text { Purine metabolism } \\
\text { Endocytosis }\end{array}$ \\
\hline & $\begin{array}{l}\text { Plant hormone signal transduction } \\
\text { Glycosphingolipid biosynthesis } \\
\text { Vitamin metabolism }\end{array}$ & $\begin{array}{l}\text { Other types of O-glycan biosynthesis } \\
\text { Purine metabolism } \\
\text { DNA replication }\end{array}$ \\
Plant-pathogen interaction & $\begin{array}{l}\text { Pyrimidine metabolism } \\
\text { Arachidonic acid metabolism }\end{array}$ \\
\hline Basal transcription factors & $\begin{array}{l}\text { Photosynthesis:antenna proteins } \\
\text { Plant hormone signal transduction } \\
\text { Plant-pathogen interaction } \\
\text { Starch and sucrose metabolism } \\
\text { Carbon fixation in photosynthetic organisms }\end{array}$ & $\begin{array}{l}\text { Purine metabolism } \\
\text { Pentose and glucuronate interconversions } \\
\text { Endocytosis } \\
\text { Pyrimidine metabolism } \\
\text { Spliceosome }\end{array}$ \\
\hline
\end{tabular}

Fig. 1 | Comparative genome profile of $\boldsymbol{M}$. viride and $\mathbf{C}$. atmophyticus. $\mathbf{a}$, This phylogenetic tree was constructed by maximum likelihood based on the concatenated sequences of single-copy genes, while species-specific gene duplicates were excluded from the analysis. A $k$-means clustering of gene families based on the gene abundance of each species is shown in the right-hand panel; each column represents a family and each row represents one species. b, Differential interference contrast micrographs showing M. viride (left) and C. atmophyticus (right). c, Venn diagrams showing the number of gene families shared among M. viride, C. atmophyticus and a representative rhodophyte, streptophyte or chlorophyte. $\mathbf{d}$, Significant increases and decreases in gene families; filled red circles, triangles and rhombi denote function enrichment of significant increased gene families in the KEGG pathway, while empty symbols denote function enrichment of significant decreased gene families in the KEGG pathway. Details of these functions are shown in the righthand panel. e, Percentages of total proteins found in both algae and embryophytes (red), proteins shared among algae (purple) and proteins shared among embryophytes (green) based on the classification given in Orthofinder. f, Principal component analysis of the type and number of Pfam domains. 


\section{Results}

Genome sequencing, genome characteristics and phylogenetic analysis. A total of $245 \mathrm{~Gb}(746.86 \mathrm{X})$ (M. viride) and $66 \mathrm{~Gb}$ (775.68X) (C. atmophyticus) raw data were generated using Illumina technology (Supplementary Tables 1 and 2). Based on $k$-mer depth distribution analyses, the nuclear genome sizes of $M$. viride and C. atmophyticus were estimated to be 329 and $85 \mathrm{Mb}$, respectively (Supplementary Tables 3 and 4 and Supplementary Figs. 1 and 2). Approximately $85.4 \%(281 \mathrm{Mb})$ and $87.0 \%(74 \mathrm{Mb})$ of the genomes were de novo assembled, consisting of 6,924 scaffolds with a minimum contig length needed to cover $50 \%$ of the genome $\left(N_{50}\right)$ of 113,221 base pairs (bp) for M. viride, and 3,836 scaffolds with $N_{50}$ of 752,385 bp for C. atmophyticus (Supplementary Tables 5 and 6). The distributions of genomic copy content (Supplementary Figs. 3 and 4) and BUSCO score (Supplementary Tables 7 and 8) suggested no contamination and good assembly quality. Moreover, $93.8 \%$ (M. viride) and $99.4 \%$ (C. atmophyticus) of de novo assembled transcripts were aligned to the assembled genome. (Supplementary Tables 9 and 10). Both genomes have substantial repeat components (38.7 and 31.3\%, respectively; Supplementary Tables 11 and 12). By combining homologue-based, ab initio and transcriptome-based approaches, 9,300 gene models were predicted for both genomes (Supplementary Tables 13 and 14 and Supplementary Fig. 5). In total, 9,198 and 9,066 predicted coding sequences, respectively, were supported by sequenced transcripts, indicating the high accuracy of gene predictions (Supplementary Table 15). Complete coverage of genes involved in transcription, translation and DNA synthesis was also obtained (Supplementary Table 16).

A phylogenetic analysis of 16 genomes from Rhodophyta, Glaucophyta, Chlorophyta, streptophyte algae and embryophytes, based on a concatenated amino acid sequence alignment of 375 orthologues of single-copy genes, confirmed the sister relationship between Mesostigma and Chlorokybus and also supported previous reports that $M$. viride and C. atmophyticus constitute the earliest-diverging lineage of extant streptophytes ${ }^{15-17}$ (Fig. 1a).

Comparative genomics. Mesostigma viride, C. atmophyticus and $K$. nitens shared 3,683 gene families (Fig. 1c), which comprised 4,423 genes in $M$. viride and 4,210 in C. atmophyticus. Interestingly, 3,639 and 2,470 gene families were found exclusive to $M$. viride and C. atmophyticus, respectively (Extended Data Fig. 1 and Supplementary Table 17). We also considered homologues of the identified genes in the red alga Cyanidioschyzon merolae, and in Micromonas commoda (Chlorophyta). M. viride and C. atmophyticus share about 2,000-2,500 gene families with C. merolae and 3,300-4,100 with M. commoda, reflecting their phylogenetic relationships (Fig. 1c).

To explore significant increases or decreases in gene families in Rhodophyta, Chlorophyta and streptophyte algae, the gene family content of five representative genomes in each of the three clades (a total of 15 genomes) was compared. The most significant increases in gene families occurred from Rhodophyta to streptophyte algae (225) and to Chlorophyta (188), respectively, and referred mainly to plant hormone signal transduction, plant-pathogen interaction, photosynthesis and starch and sucrose metabolism (Fig. 1d). Conversely, significant decreases in gene families were observed from the two lineages of Viridiplantae to Rhodophyta (41 and 44, respectively), mainly corresponding to purine metabolism, endocytosis and pentose and glucuronate interconversions. In comparison, significant increases and decreases in gene families between streptophyte algae and Chlorophyta were more modest: the main increases for gene families in streptophyte algae were in plant hormone signal transduction, plant-pathogen interactions and TFs, whereas the main decreases for gene families were in purine and pyrimidine metabolism, DNA replication and O-glycan biosynthesis.

In addition, our analysis revealed a higher percentage of embryophyte genes in $M$. viride and C. atmophyticus compared to Chlorophyta, but lower than in $K$. nitens (Fig. 1e). In addition to gene families, the total number of conserved Pfam domains in Rhodophyta, Chlorophyta and Streptophyta was subjected to principal component analysis, which showed distinct patterns of functional diversification indicating evolutionary diversification (Fig. 1f). Overall, these results suggest that the genomes of earlydiverging streptophyte algae already contained archetypal genes that typically exist in modern embryophytes.

TFs and phytohormones in early-diverging Streptophyta. Out of 114 types of TFs/transcription regulators (TRs) analysed, 72 (M. viride) and 80 (C. atmophyticus) TFs/TRs were identified in the two genomes (Fig. 2a and Supplementary Table 18), of which most are associated with abiotic stress responses, development and plant-pathogen interactions in embryophytes. TF/TR genes accounted for $3.31 \%$ of the total number of protein-coding genes in the $M$. viride genome and $4.47 \%$ in the C. atmophyticus genome, similar to the percentages in bryophytes (4.68\%) and angiosperms $(\sim 5 \%)$. A combination of HMMER and phylogenetic analyses (for details see Methods) revealed putative gains of several TF/TR genes in the common ancestor of streptophytes, and differential losses of some of these genes in M. viride and/or C. atmophyticus (Fig. 2b). We cannot, however, exclude the possibility that the lower TF/TR numbers in $M$. viride relate to lack of representation in the genome assembly, although we aimed to compensate for this by adding data from our deeply sequenced transcriptome. Among the gains, the homeodomain-leucine zipper (HD-ZIP) family members are unique to plants and have diverse functions in growth and development, mostly related to stress response ${ }^{19}$. Of the four known classes (I-IV) of HD-ZIPs, gene classes I-III were found in C. atmophyticus but were missing in $M$. viride (Fig. 2a and Supplementary Fig. 6). $M$. viride displayed one gene that was positioned near the base of the HD-ZIPIV clade in phylogenetic analyses with moderate support and a long branch (Supplementary Fig. 6). However, this gene contained neither a HD nor a START domain, suggesting that it represents either an ancestral pre-HD-ZIP_IV or a degenerated gene with domain, and perhaps functional, loss. Other TF/TR genes that apparently originated in the common ancestor of streptophytes, such as auxin response factors (ARF) proto-C-type (Fig. $2 b$ and Supplementary Figs. 7 and 8), SHI-related sequence (SRS), Trihelix TF family (Trihelix), growth-regulating factors (GRF), LUG transcriptional co-repressor (LEUNIG) and HD-PLINC homeodomain plant zinc finger (HD-PLINC), have also been implicated in the regulation of growth and development in response to light, salt and pathogen stresses in embryophytes ${ }^{20-25}$. In addition to gains, nine TF/TR gene families expanded by a minimum of threefold in $M$. viride/C. atmophyticus compared to non-streptophytes (Supplementary Table 18), and five of those (C2C2_CO-like, DDT, PcG_FIE, PcG_MSI, PSEUDO-ARR-B) had a minimum of $50 \%$ more members in C. atmophyticus than in M. viride, all involved in responses to abiotic and biotic stresses in embryophytes. Overall, our results correlate well with a recent analysis using transcriptome data $^{13}$, except that we extend the origin of HD-Zip genes I-III, ARF and HD-KNOX1 (Knotted-like homebox class I) to the common ancestor of streptophytes rather than a later origin.

Next, we explored phytohormone biosynthesis and transduction pathway-related genes (Fig. 2c and Supplementary Tables 19 and 20). By employing a HMMER search, an almost intact abscisic acid (ABA) signalling pathway was detected although the ABA receptor PYL was absent, the latter finding consistent with earlier reports on $K$. nitens ${ }^{6}$ and C. braunii ${ }^{7}$. Homologues of the Abscisic acid responsive element-binding protein (AREB)/Abscisic acid responsive element-binding factors (ABFs) TFs involved in ABA signalling under drought stress in embyophytes were also detected in both genomes ${ }^{26}$. Genes for the complete signalling pathway of cytokinin $\left(\mathrm{CK}^{27}\right)$ were identified in both genomes. The second, 


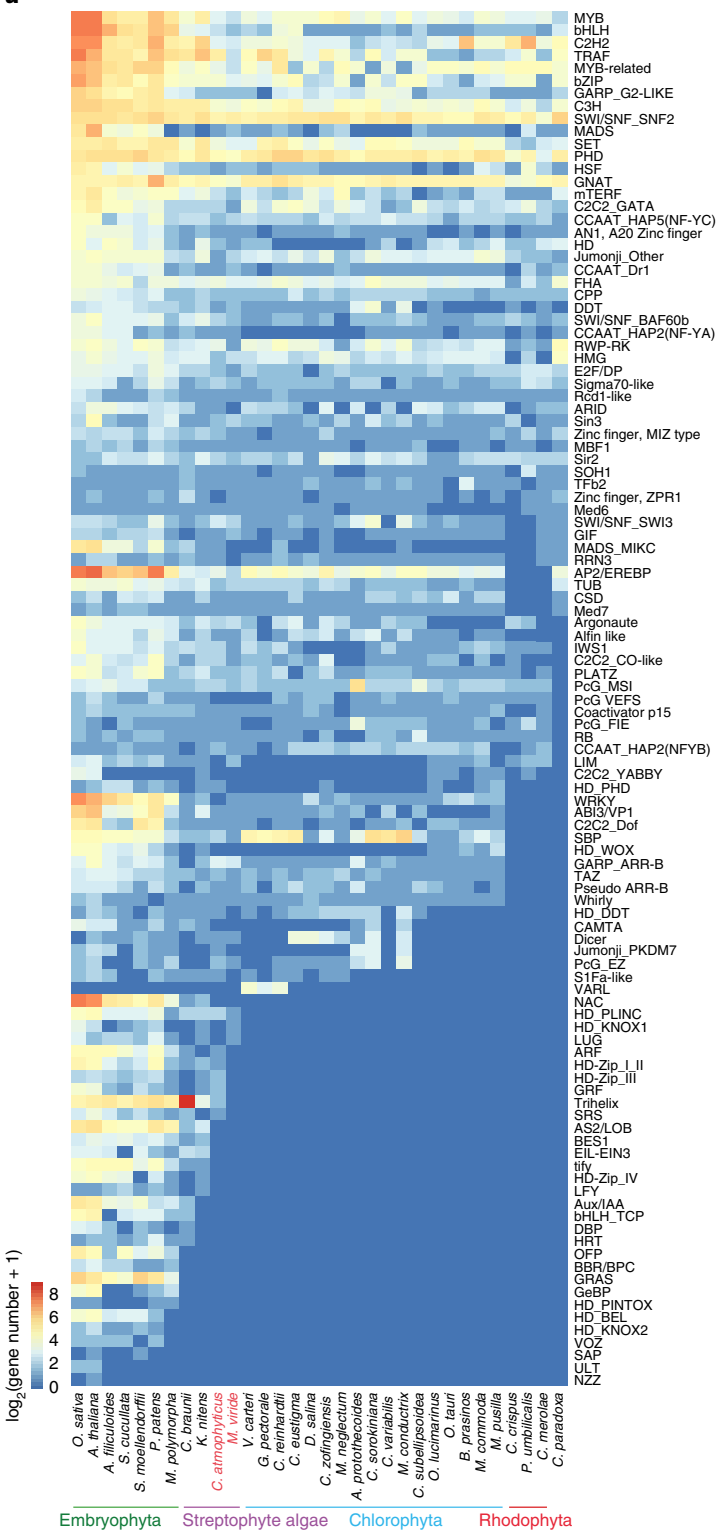

b

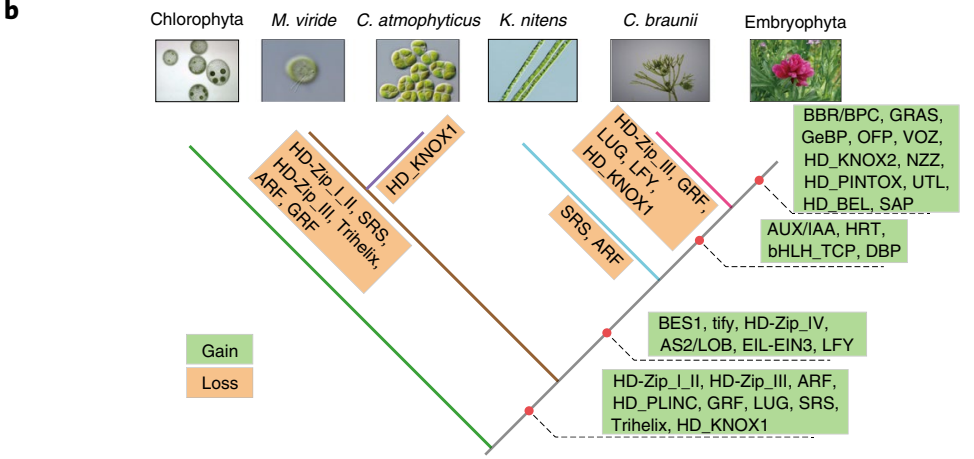

c

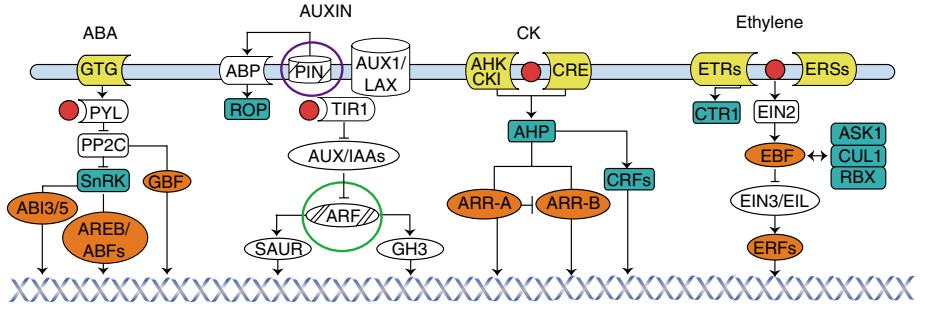

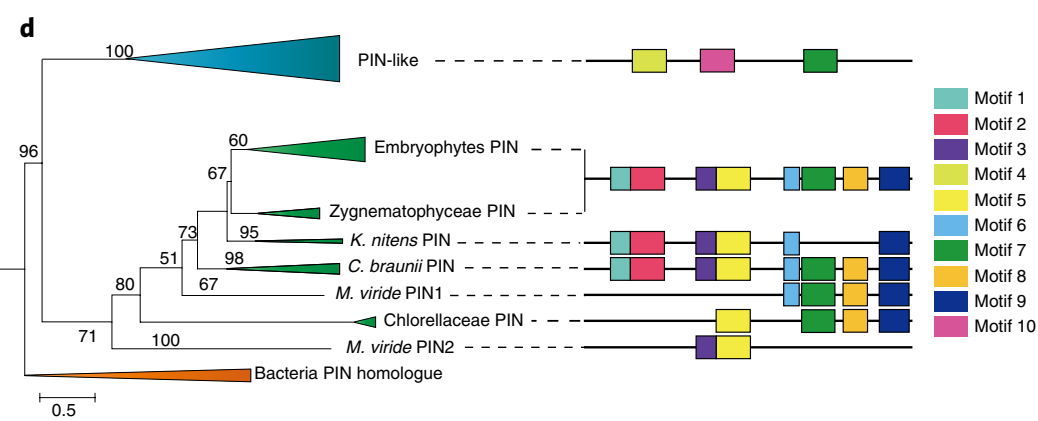

Fig. 2 | Analysis of TF genes and phytohormone signalling pathways in $\mathbf{M}$. viride and C. atmophyticus. a, Using a HMMER approach for the respective genomes, the numbers of TFs and TRs were identified using the TAPscan database v.2 (for details see Methods). b, Illustrative phylogenetic representation of the predicted gain (green) and loss (orange) of plant TFs in streptophyte algae. $\mathbf{c}$, Presence/absence of the main phytohormone signalling pathways deduced from the genomes of $M$. viride and $C$. atmophyticus. Coloured boxes indicate the presence of genes in the pathways, white boxes their absence. All searches were done using HMM $\left(1 \times 10^{-10}\right)$. Purple-lined ellipses denote genes identified in $M$. viride but not in $C$. atmophyticus, while the green-lined ellipse denotes a gene identified in C. atmophyticus but not in M. viride. d, The maximum-likelihood method was used to draw the phylogenetic tree of the PIN and PIN-related homologues to understand their origin among Streptophyta.

conserved, two-component signalling pathway (in addition to CK), that of ethylene, was also almost completely represented except for the TF EIN3/EIL.

Both M. viride and C. atmophyticus were found to encode various transporter proteins and ATP-binding cassette B transporters that could potentially participate in auxin transport (Supplementary Table 21). However, no auxin receptor (TIR) was found in either genome (Fig. 2c). Notably, PIN, an important auxin efflux carrier involved in the transport of auxin between cells, was detected in the $M$. viride genome but not in C. atmophyticus. We found a step-by-step gain in motifs corresponding to PIN (Fig. 2d). Neither M. viride nor C. atmophyticus encodes the auxin signal transduction components AUX/IAA1, SAUR and GH3 (Fig. 2c).
Furthermore, both genomes lacked auxin biosynthesis genes such as tryptophan-aminotransferase (TAA) and nitrilase, while YUCCA could be identified in $M$. viride only (Supplementary Table 19 and Supplementary Fig. 9).

Both organisms could synthesize most of the jasmonic acid (JA)-precursor OPDA but lacked OPR3, indicating that the JA biosynthetic pathway may exist in early-diverging streptophyte algae (Supplementary Table 19). Concomitantly, both C. atmophyticus and $M$. viride lacked the complete JA and strigolactone (SL)) signalling components and the respective hormone receptors (Fig. 2c). Gibberellic acid (GA) receptors (GID1 and GID2) and signalling components (DELLA, PIL1 and PIF) were also absent (for the PIF and bHLH phylogeny, see Supplementary Figs. 10 and 11). Some 
components of the salicylic acid (SA) pathway (CUL3 and TGA) were, however, detected (for TGA see Supplementary Figs 12 and 13). Finally, we analysed sequence conservation of phytohormone receptor genes among representative algal lineages (Extended Data Fig. 2). The results showed that, although the genomes of $M$. viride and C. atmophyticus encode some phytohormone-related receptors, they are not as well conserved as in embryophytes. In general, our results are in accordance with previous genome analyses of laterdiverging streptophyte algae and liverworts ${ }^{5-8}$ which suggested that F-box-mediated phytohormone signalling pathways (auxin, JA, GA, $\mathrm{SL})$ evolved in the embryophyte ancestor, whereas two-component system phytohormone signalling ( $\mathrm{CK}$, ethylene) evolved much earlier, having been present in the common ancestor of streptophytes (this study). Furthermore, some components of both F-boxmediated (auxin, SA) and other phytohormone signalling pathways (ABA, brassinosteroid (BR)) are ancient and presumably originated in the common ancestor of streptophytes-for example, PIN, C-type ARF and TGA (this study).

Analysis of cell wall metabolism, evidence of sexual reproduction and analysis of flagellar genes. In total, 81 and 100 putative carbohydrate-active enzymes (CAZymes) and 20 and 22 additional proteins containing putative carbohydrate-binding modules were identified in $M$. viride and $C$. atmophyticus, respectively (Supplementary Table 22). Both the numbers of glycosyl hydrolases (GH) and glycosyltransferases (GT) were higher in C. atmophyticus than in $M$. viride, but in the same range as those found in unicellular red algae (32/54) or early-diverging Chlorophyta ${ }^{28}(52 / 57 / 77)$. Compared to previous transcriptome-based analyses ${ }^{12}$, the differences in numbers of GTs between $M$. viride and C. atmophyticus were not as large, suggesting that hidden life history stages in $M$. viride (zygotes?) may contribute GTs to its overall genomic GT complement. The largest GT families found were GT2 (N-glycosylation and cell wall biosynthesis), GT5 (starch synthases) and GT35 (glycogen/starch phosphorylases). However, certain differences were also encountered: GT 77 (involved in rhamnogalacturonan-II synthesis) had more family members in C. atmophyticus $^{29}$, while GT 41 ( $\beta-N$-acetylglucosaminyltransferase) was more prominent in $M$. viride. Four GT families were present in only M. viride or C. atmophyticus, among them GT8 (in C. atmophyticus), a large family of glycosyltransferases in embryophytes, of which some members have been implicated in responses to abiotic stress ${ }^{30}$.

To gain further insight into the evolution of proteins involved in cell wall biosynthesis, we analysed the phylogeny of representative enzymes. Our analyses revealed the presence of three putative cellulose synthase-like (CSL) enzymes (CSLA/CSLC-like), but the absence of cellulose synthase (CESA), in M. viride (Fig. 3a and Supplementary Table 23). However, three CESA/CSLD-like homologues were identified in the C. atmophyticus genome and showed high similarity to the respective genes in embryophytes. C. atmophyticus displayed two additional CSL enzymes that apparently originated by a gene duplication in the common ancestor of $M$. viride and C. atmophyticus (Fig. 3a). Our phylogenetic analysis demonstrated that CESA and CSL proteins diverged into two clades, their origin apparently dating back to the ancestor of the Archaeplastida ${ }^{31}$. The first clade encompassed CESA and CLSB, -D, -E, -G and - H, the second clade CSLA, CSLC and a paraphyletic set of genes (CSLA/ CSLC-like) that includes five CSL genes from M. viride and C. atmophyticus. Mapping CESA and CSLs on a simplified phylogenetic tree of Archaeplastida pinpoints putative gains and losses of these enzymes on different branches (Fig. 3b).

To adapt to rapidly changing environments, early-diverging streptophytes would be expected to actively conduct reassembly and degradation of cell wall components to enhance the flexibility of the cell wall during osmotic stress. Enzymes in 14 families of glycoside hydrolases (GH5-10,-12, -26, -44,-45, -48, -51, -61 and -74) are known to degrade cellulose in embryophytes ${ }^{32}$. Not surprisingly, cellulases were not detected in the $M$. viride genome, which lacks CESA; however, in C. atmophyticus cellulases were present (Table 1). Interestingly, a majority of the genes involved in mannan and xylan metabolism, such as mannanases, mannosidase and xylosidase, were detected in $M$. viride whereas $C$. atmophyticus lacks those enzymes. $M$. viride and C. atmophyticus also lack xyloglucan- and xylandegrading enzymes, as well as most of the pectin lyases (Table 1).

Some of the differences in the composition of the cell surface between $M$. viride and C. atmophyticus could be related to variation in their cellular organization, life history and habitats. To check whether 'cryptic sex' may exist in early-diverging streptophyte algae, we searched the $M$. viride and C. atmophyticus genomes for meiosis-specific (11) and meiosis-related genes (40) by hidden Markov model (HMM) and BLAST (Supplementary Table 24). We found that the core-set of meiosis-specific genes (10) was present in C. atmophyticus whereas the M. viride genome lacked MSH5, REC8 and RED1 (Supplementary Table 24); the absence of the latter is puzzling, but not without precedent ${ }^{33}$. M. viride reproduces vegetatively by binary division of the flagellate cell, whereas C. atmophyticus forms scale-covered zoospores during asexual reproduction. A comparative analysis of the complement of flagellar genes in their genomes, using a stringent reciprocal-best-BLAST-hits analysis of 397 Chlamydomonas flagellar proteins as query ${ }^{34}$, detected 204 flagellar proteins in M. viride and 192 in C. atmophyticus (Fig. 4 and Supplementary Table 25). Non-flagellate organisms lack the majority of radial spoke, as well as central pair proteins, many of the outer and inner dynein arm proteins and all intraflagellar transport proteins (IFTs), as well as dynein heavy-chain proteins. Surprisingly, in both $M$. viride and C. atmophyticus (flagella covered by scales), we could identify only a few IFTs whereas K. nitens displayed the full set (12 IFTs) (Extended Data Fig. 3).

Evolutionary analysis of elongation factor- $1 \alpha$ and phytochromes. The elongation factor EF- $1 \alpha$ is responsible for the selection and binding of aminoacyl-transfer RNA to the A-site (acceptor site) of the ribosome. It is substituted by elongation factor-like (EF-like) proteins in many eukaryotes ${ }^{35}$. Intriguingly, we found that the $M$. viride genome encodes both EF- $1 \alpha$ and EF-like genes (Fig. $5 \mathrm{a}$ and Extended Data Fig. 4), contradicting earlier studies that reported only the presence of EF-like genes in M. viride and no EF- $1 \alpha$ (ref. ${ }^{36}$ ).

The origin of the canonical embryophyte phytochrome ( $\mathrm{p}-\mathrm{PHY}$ ) can be traced to the ancestor of extant streptophyte algae ${ }^{37}$. Two phytochrome genes were identified in both the $M$. viride and C. atmophyticus genome, which is in accordance with a previous transcriptome study ${ }^{37}$ (Extended Data Fig. 5). Interestingly, the two genes of $M$. viride showed differing domain structures, one gene containing a response regulator at the Cterminus regulatory module (REC), which was not identified in the previous transcriptome study ${ }^{37}$. Phylogenetic analyses indicated that a gene duplication occurred in the ancestor of streptophytes, followed by loss of REC in one of the duplicated genes, resulting in the evolution of $\mathrm{p}$-PHY (Fig. 5b). Non-canonical phytochromes (PHYX1 and PHYX2) were retained in several streptophyte algal lineages.

\section{Discussion}

The successful colonization of the terrestrial landscape by plants, and their subsequent rapid evolution, is considered to be a pivotal event in the evolution of life. Here, we present the draft genomes of two early-diverging streptophyte algae that thrive in contrasting habitats: $M$. viride is found in the benthos of small shallow ponds, whereas $C$. atmophyticus is a subaerial/terrestrial alga. All previous phylogenomic analyses, including the present study, placed the two species as sister taxa in the earliest-diverging clade of streptophytes ${ }^{14-17}$. Their contrasting habitats and phylogenetic position make the genomes of $M$. viride and C. atmophyticus an 

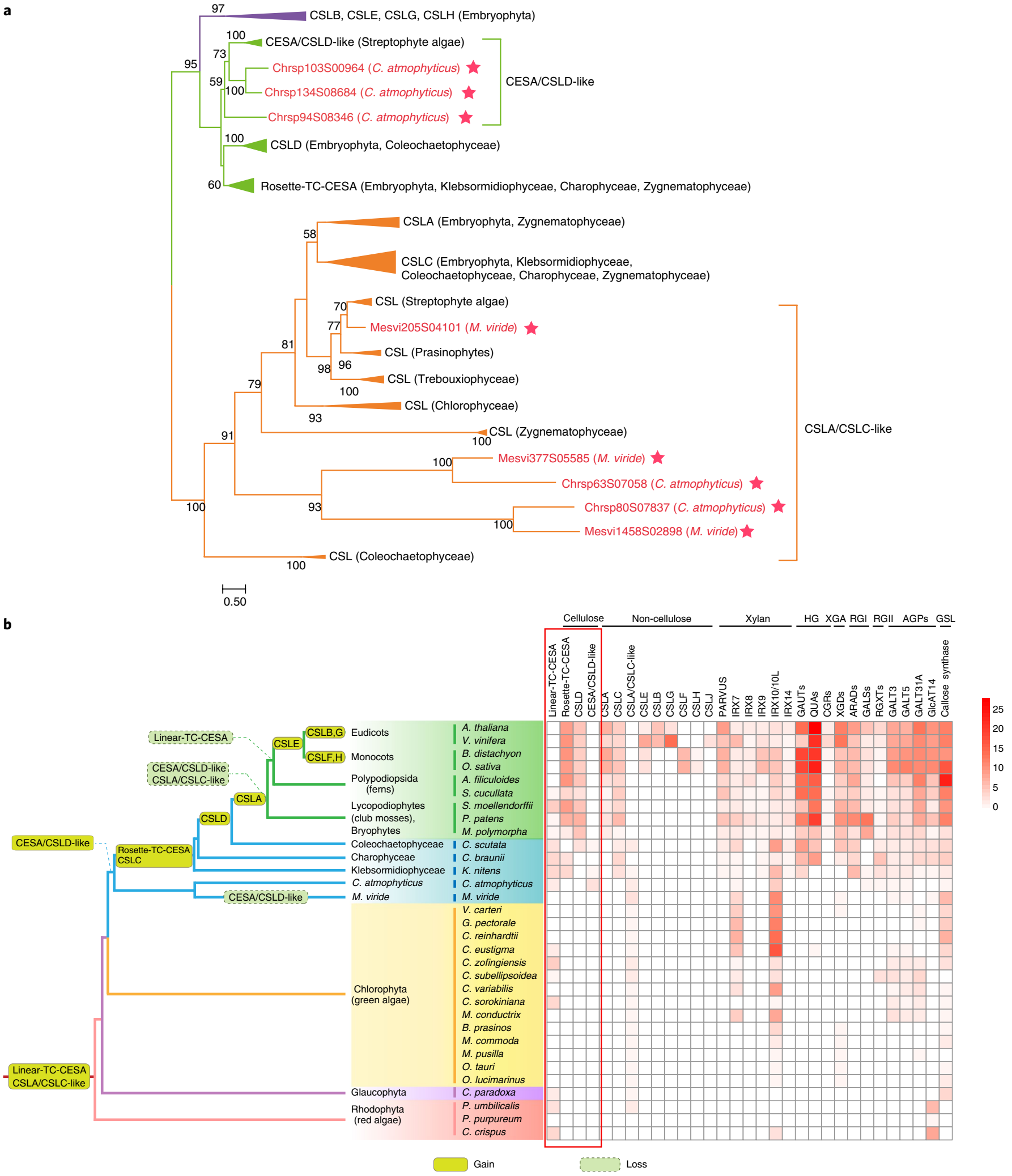

Fig. 3 | Phylogenetic distribution of enzymes involved in cell wall biosynthesis in $\boldsymbol{M}$. viride and C. atmophyticus. a, Phylogenetic tree of CESA and CSL (GT2 family). The tree was constructed using the maximum-likelihood method. $\mathbf{b}$, Left: summary of the gains and losses of cell wall biosynthetic genes mapped on the phylogenetic tree. Right: copy number of the respective cell wall biosynthetic genes. HG, homogalacturonan; XGA, xylogalacturonan; RGI and RGII, rhamnogalacturonan I and II; AGP, arabinogalactan protein; GSL, glucan synthase-like.

exciting resource for comparative investigations into land plant evolution. Our genome analyses suggest that early-diverging streptophyte algae already took the first step on the long road to plant terrestrialization and harbour many embryophyte-type genes. Compared to Chlorophyta, the main gene family gains and expansions in early-diverging streptophyte algae were in plant 


\begin{tabular}{|c|c|c|c|c|}
\hline & Enzyme & M. viride & C. atmophyticus & K. nitens \\
\hline Cellulase & $\begin{array}{l}\text { Endoglucanase (GH5-10, -12, }-26,-44,-45,-48,-51,-61 \text {, } \\
-74 \text { ) }\end{array}$ & 0 & 3 & 8 \\
\hline \multirow[t]{5}{*}{ Glycosidase } & $\mathrm{GH} 27$ ( $\alpha$-galactosidase $)$ & 1 & 2 & 5 \\
\hline & GH35 ( $\beta$-galactosidase) & 1 & 2 & 2 \\
\hline & GH36 (galactinol-sucrose galactosyltransferase) & 0 & 2 & 3 \\
\hline & GH37 (glucan endo-1,3- $\beta$-glucosidase) & 0 & 0 & 9 \\
\hline & $\mathrm{GH} 1$ ( $\beta$-glucosidase) & 1 & 1 & 5 \\
\hline \multirow[t]{2}{*}{ Xylosidase } & GH31 ( $\alpha$-xylosidase) & 0 & 2 & 0 \\
\hline & GH3 ( $\beta$-D-xylosidase) & 1 & 1 & 2 \\
\hline \multirow[t]{2}{*}{ Xyloglucan } & GH12 (xyloglucan endotransglucosylase/hydrolase) & 0 & 0 & 0 \\
\hline & GH16 (xyloglucan endotransglucosylase) & 0 & 0 & 5 \\
\hline Xylanases & GH10 (1,4- $\beta$-xylan endohydrolase) & 1 & 0 & 9 \\
\hline Xyloglucanase & GH77 (xyloglucanase) & 0 & 4 & 3 \\
\hline \multirow[t]{2}{*}{ Arabinofuranosidase } & GH43 (arabinanase) & 0 & 0 & 1 \\
\hline & GH51 ( $\alpha$-L-arabinofuranosidase) & 1 & 1 & 1 \\
\hline \multirow[t]{4}{*}{ Pectin lyases } & Pectate lyase & 0 & 0 & 1 \\
\hline & Rhamnogalacturonate lyase & 0 & 0 & 1 \\
\hline & Pectinesterase & 0 & 0 & 1 \\
\hline & Pectin acetylesterase & 0 & 1 & 1 \\
\hline Mannosidase/mannanase & GH5 (mannan endo-1,4- $\beta$-mannosidase) & 2 & 0 & 5 \\
\hline
\end{tabular}

hormone signal transduction, plant-pathogen interactions and TFs and regulators, most related to environmental stresses (light, temperature, salt, drought and pathogens) that are thought to have played a major role in plant terrestrialization.

Mesostigma viride and C. atmophyticus differ distinctively in their life history and cellular organization. To test whether these differences between the two species are responsible for their genome differences, we analysed the complement of flagellar genes. We detected 204 of 397 Chlamydomonas flagellar proteins in the $M$. viride genome. Similar numbers of conserved flagellar proteins exist in streptophyte algae that produce reproductive flagellate cells (zoospores, spermatozoids) during their life history (240 in $K$. nitens and 209 in C. braunii), suggesting that the ancestral number of flagellar proteins in Streptophyta was 200-250. Second, we looked for possible differences in their life histories focusing on sexual reproduction (in C. atmophyticus sexual reproduction is unknown, in $M$. viride only a preliminary report exists). The coreset of meiosis-specific genes (10) were present in C. atmophyticus, whereas the $M$. viride genome lacked three of them. This suggested that both species reproduce sexually, although the process itself remains poorly understood.

Further insight into the differences in gene composition between $M$. viride and C. atmophyticus was obtained by focusing on genes involved in adaptations to the different habitats in which both species thrive. With the streptophyte algal phylogeny basically resolved, we mapped TF/TR genes on the phylogenetic tree and determined putative gains, losses, expansions and contractions of TF/TR gene family members. From this, we conclude that TFs/TRs that regulate growth and development in responses to light, salt and pathogen stresses in embryophytes originated in the common ancestor of streptophytes. Interestingly, some of the TFs/TRs thought to be involved in responses to light, salt and pathogen stresses, such as the HD-ZIPs_I-II, SRS and GRF, were absent in M. viride, perhaps lost in relation to its aquatic habitat. This hypothesis is consistent with the previous genome analysis of $C$. braunii (a structurally complex, streptophyte alga from an aquatic, benthic habitat) that also seems to have lost an HD-ZIP gene (HD-ZIP_IV), another HD-gene (HD_KNOX 1) and $\mathrm{GRF}^{7}$; HD-ZIP genes have also been lost in some secondarily aquatic embryophytes ${ }^{38}$.

Although elements of several biosynthetic and signalling phytohormone pathways were identified in $M$. viride and C. atmophyticus, they were mostly incomplete and only the CK pathway, considered to be involved in abiotic stress responses ${ }^{39}$, was fully recovered. Ethylene-related signalling homologues have previously been reported in Spirogyra pratensis and Coleochaete orbicularis $^{40,41}$, and this is consistent with our findings. K. nitens also displays most of the ethylene-related genes, including the TF EIN3/ EIL which is lacking in M. viride and C. atmophyticus, suggesting that this TF had its origin in the common ancestor of $K$. nitens and derived streptophytes ${ }^{6}$. The ABA pathway was also nearly complete but lacked the receptor PYL. A PYL orthologue was recently identified in a transcriptomic analysis of the streptophyte alga Zygnema (Zygnematophyceae). The absence of the F-boxmediated AUXIN, JA, GA and SL signalling pathways in the earlydiverging streptophyte algae is not surprising, as previous studies have reported orthologues of most of the signalling components of these pathways in genomes of embryophytes but not of algae $e^{5-7,42,43}$. Interestingly, we identified the origin of several signalling components involved in phytohormone pathways. The PIN protein, an important auxin efflux carrier ${ }^{44}$, seems to have originated in the common ancestor of streptophytes (two PINs were detected in $M$. viride) or even in the common ancestor of Viridiplantae (contrary to a previous study, we detected the presence of PIN also in the Chlorellaceae ${ }^{45}$ (Chlorophyta)).

Previous studies have shown that the most important core cell wall polysaccharides of embryophytes, namely cellulose, mannan, xyloglucan, xylan and pectin, are also represented in diverse lineages of streptophyte algae ${ }^{12}$. The cellulose synthase-like gene families are among the most important players involved in the formation of plant cell walls ${ }^{46}$. Three CESA/CSLD-like homologues were identified in the C. atmophyticus genome, which showed high similarity to the respective genes in embryophytes. Our analyses also revealed 


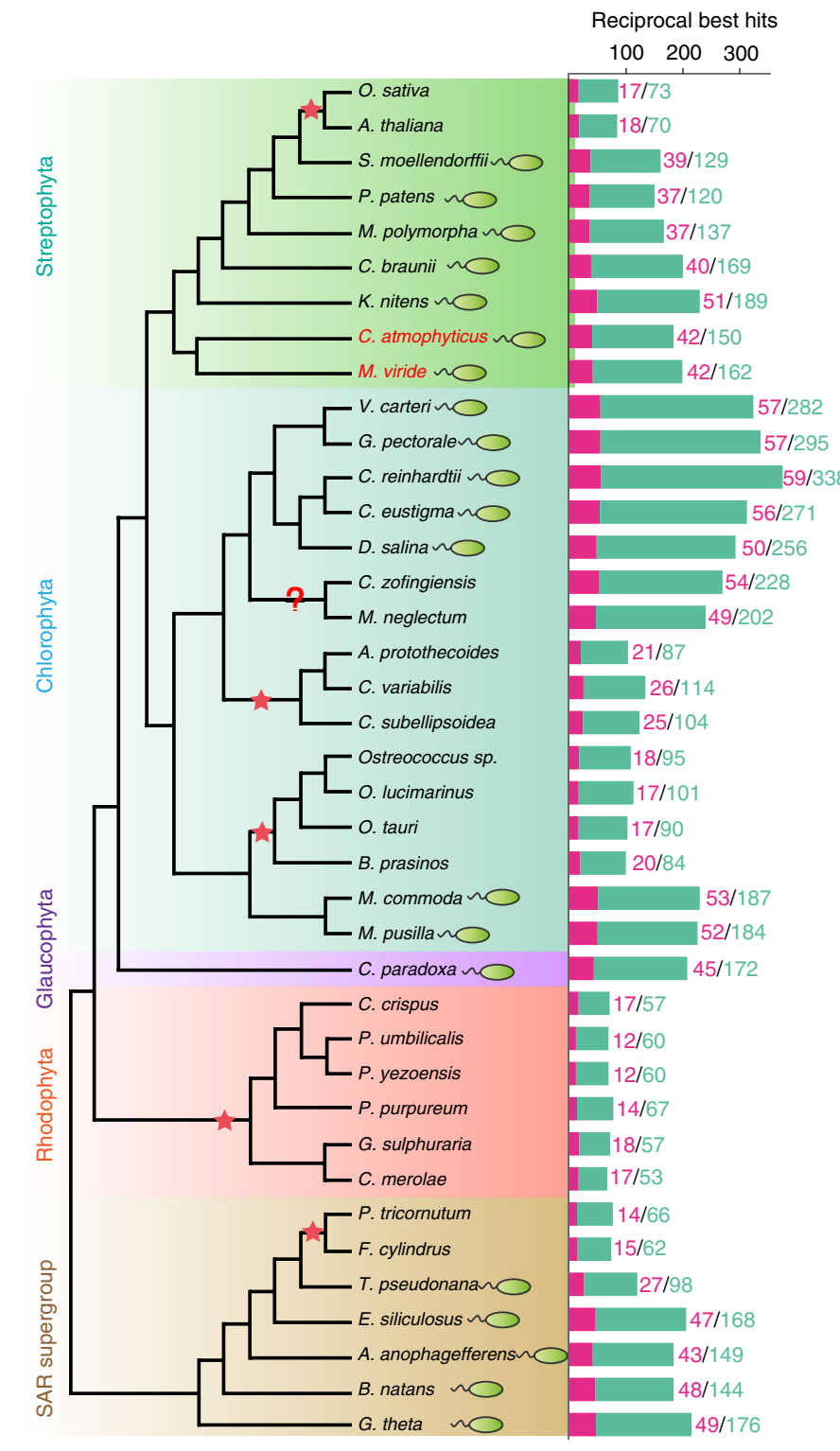

Flagellum loss events
Central pair Radial Spoke Protein Protein Outer dynein Inner dynein Protein Dynein protein

Selected flagellar proteins

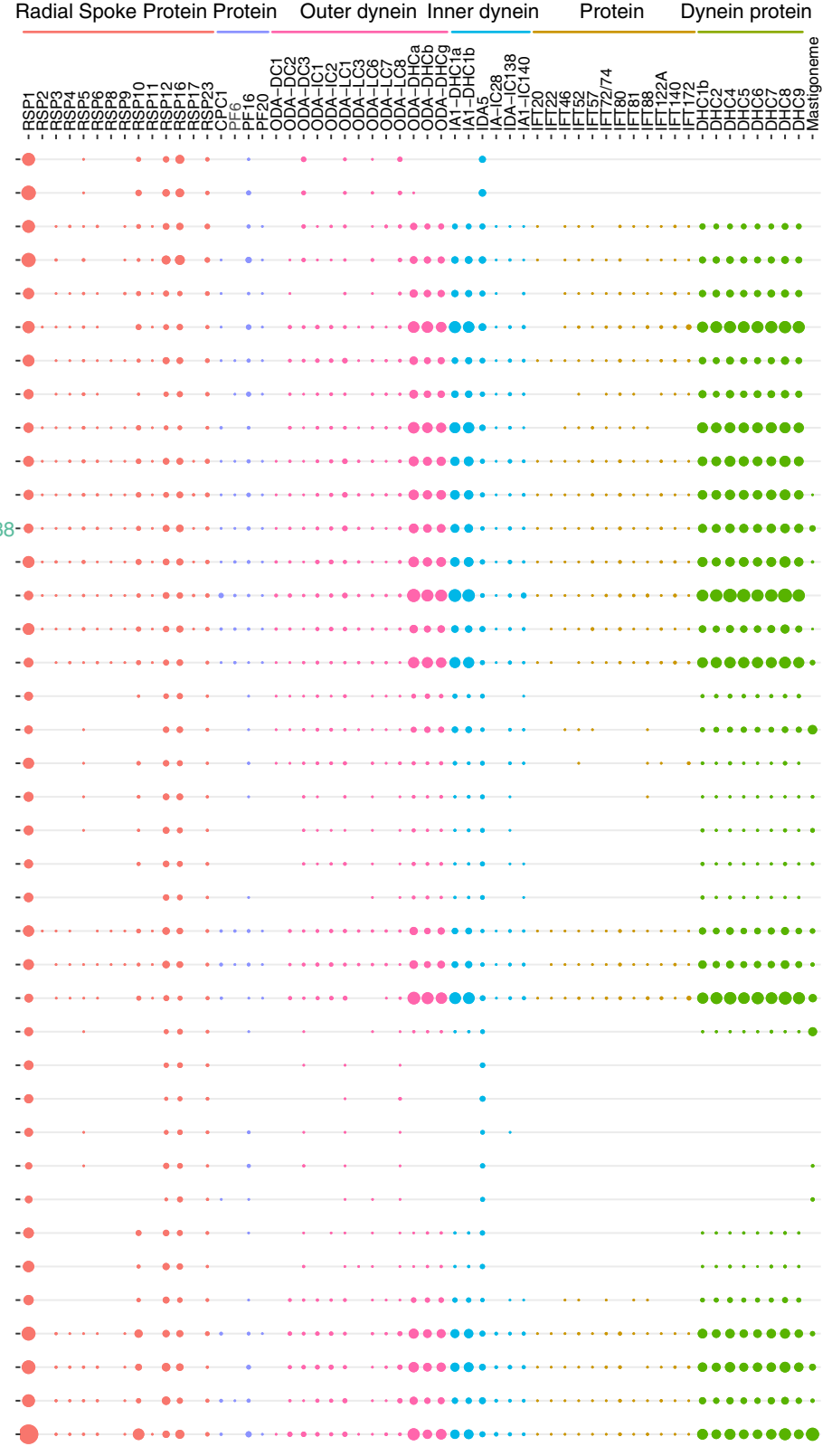

Other flagellar proteins ? Unknown

Fig. 4 | Analysis of flagellar genes and their phylogenetic distribution. The phylogenomic tree (left) was constructed using a maximum-likelihood method based on the concatenated sequences of single-copy genes from different representative algal lineages. The horizontal bar chart (middle) denotes the number of putative orthologues to 398 Chlamydomonas conserved flagellar proteins; the pink horizontal bar represents the number of structure-related flagellar genes (individual genes listed at the top of the right panel), while the green area represents the number of flagella-associated genes. The right panel shows the key structure-related flagellar proteins in six categories. The circle size is proportional to the copy number of putative orthologous genes found in the respective species.

the presence of putative CSL enzymes (CSLA/CSLC-like) in both genomes, but no CESA in $M$. viride, the latter corroborating a previous analysis using expressed sequence tags ${ }^{47}$. Our phylogenetic analysis indicated that the CESAs and CSLDs of embryophytes were derived from CESA/CSLD-like homologues present in the common ancestor of streptophytes that were lost in $M$. viride.

Moreover, homologues of biosynthetic genes for Rhamnogalacturonan-I and -II (RG), which are considered among the evolutionarily youngest cell wall polysaccharides of embryophytes $^{12}$, such as RGXT and GALSs, were also identified in C. atmophyticus but not in M. viride. We also found cellulases in both C. atmophyticus and K. nitens but not in M. viride, indicating that $M$. viride lost these enzymes together with cellulose when adapting to a flagellate life history and an aquatic habitat. It is likely that mannan performs the function of cellulose in $M$. viride and may be restricted to a putative zygotic stage. Xyloglucan and xylan presumably evolved later (in either the common ancestor of $K$. nitens and other streptophytes or in the ancestor of derived streptophyte algae $\mathrm{e}^{12,48,49}$ ). In summary, $M$. viride and C. atmophyticus differ considerably in their CAZymes, as expected from variation in their cellular organization, life history and habitats, but also from later-diverging streptophyte algae such as K. nitens. 

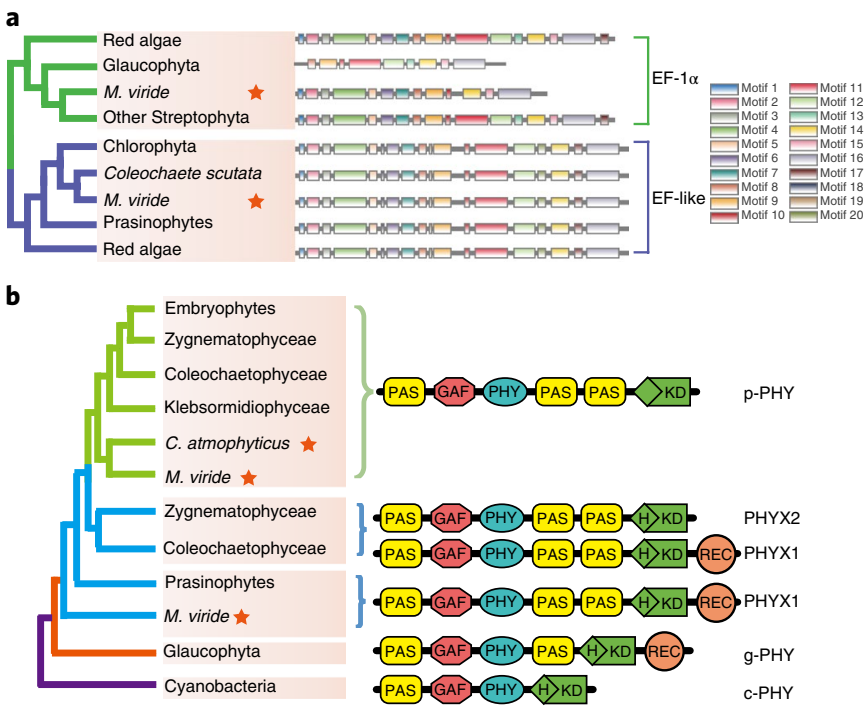

Fig. 5 | Distribution of EF-1 $\alpha$, EF-like and plant phytochromes.

a, Maximum likelihood was used to infer the phylogenetic tree. Right: representative EF-1 $\alpha$ and EF-like motifs, from red algae to embryophytes. 'Other Streptophyta' represents C. atmophyticus, Klebsormidiophyceae, Coleochaetophyceae, Charophyceae and Zygnematophyceae. b, Left: simplified phylogenic tree of phytochromes across cyanobacteria, glaucophytes, prasinophytes and streptophyte algae is shown. Right: complete domain structures of the phytochrome proteins. PHYX1/PHYX2 represent the sister lineage to $\mathrm{p}-\mathrm{PHY}$. The phytochromes from glaucophytes and cyanobacteria are represented by $\mathrm{g}-\mathrm{PHY}$ and c- $\mathrm{PHY}$, respectively.

Our analyses of phytochromes in the genomes of M. viride and C. atmophyticus corroborate previous transcriptome studies ${ }^{37}$, in that the origin of the canonical embryophyte phytochrome (p-PHY) can be traced to the common ancestor of streptophytes. We tentatively identified a gene duplication event in the common ancestor of streptophytes, followed by loss of the response regulator (REC) and the histidine phosphorylation site $(\mathrm{H})$ in one of the duplicated genes. Notably, both phytochrome genes of $C$. atmophyticus clustered with canonical embryophyte phytochromes (p-PHY) as sisters of p-PHY of $M$. viride.

\section{Conclusions}

Two major conclusions can be drawn from the comparative analysis of the draft genomes of M. viride and C. atmophyticus: first, the common ancestor of M. viride and C. atmophyticus had already developed traits that reflect adaptations to a subaerial/terrestrial habitat, exemplified by the presence of the canonical embryophyte photoreceptor phytochrome (p-PHY), evolution of TFs implicated in responses to various abiotic and biotic stresses, near-complete pathways for several phytohormones involved in stress signalling and evolution of orthologues of cellulose synthase and cellulose synthase-like genes characteristic of embryophytes. The common ancestor of streptophytes had thus taken the first step toward plant terrestrialization, supporting a recent hypothesis that streptophyte algae lived on land before the emergence of embryophytes ${ }^{50}$.

Second, the genomes of $M$. viride and C. atmophyticus differ conspicuously in genome size, structure and gene complement, revealing the dynamic nature of their genomes perhaps in response to adaptations to their contrasting habitats. Furthermore, the phylogenetic relationship of $M$. viride and C. atmophyticus as sister taxa leads to the conclusion that $M$. viride lost several traits of a subaer$\mathrm{ial} /$ terrestrial ancestry following transition to a benthic, freshwater habitat, a situation that finds a parallel in the secondarily aquatic C. braunii ${ }^{7}$. Although adaptations to terrestrial life progressed stepwise in streptophyte algae, this did not follow a linear path with deviations into aquatic habitats apparently occurring repeatedly, requiring careful comparative genomic and phylogenomic analyses of a larger taxon set of streptophyte algae in future studies.

\section{Methods}

Culture, nucleic acid extraction and light microscopy. Axenic cultures of M. viride (CCAC 1140) and C. atmophyticus (CCAC 0220) were obtained from the Culture Collection of Algae at the University of Cologne, and grown in Waris- $\mathrm{H}$ culture medium ${ }^{51}$ (http://www.ccac.uni-koeln.de/). During all steps of culture scale-up until nucleic acid extraction, axenicity was monitored by both sterility tests and light microscopy. Total RNA was extracted from $M$. viride using the Tri Reagent Method, and from C. atmophyticus using the CTAB-PVP method as described in ref. ${ }^{52}$. Total DNA was extracted using a modified CTAB protocol ${ }^{53}$ (details below). Light microscopy was performed with a Leica DMLB light microscope using a PL-APO $\times 100 / 1.40$ numerical aperture (NA) objective, an immersed condenser (NA, 1.4) and a Metz Mecablitz 32 Ct3 flash system.

Genome sequencing, data preparation and genome assembly. Paired-end libraries with insert sizes of $170 \mathrm{bp}, 200 \mathrm{bp}, 500 \mathrm{bp}, 800 \mathrm{bp}, 2 \mathrm{~kb}, 5 \mathrm{~kb}, 10 \mathrm{~kb}$ and $20 \mathrm{~kb}$ were constructed following standard Illumina protocols. The libraries were sequenced on an Illumina HiSeq 2000/4000 and BGI-seq 500 platform. A total of $245 \mathrm{~Gb}$ (about 746.86X) and $66.46 \mathrm{~Gb}$ (about 775.68X) paired-end data were generated for M. viride (CCAC 1140) and C. atmophyticus (CCAC 0220), respectively. To reduce the effect of sequencing error on assembly, we performed a rigorous quality control of raw data. We used CLC Assembly Cell (v.5.0.1) ${ }^{54}$ to trim the adaptors, remove duplicates and trim low-quality bases. We then used Pairfq (v.0.16.0) (https://github.com/sestaton/Pairfq) to pair the reads. Finally, SOAPfilter (v.2.2) was used to filter the reads again. After filtering off duplicated and lowquality reads and those with adaptor sequences, 74.63 and $14.51 \mathrm{~Gb}$ high-quality clean reads remained for M. viride (CCAC1140) and C. atmophyticus (CCAC 0220), respectively, which were then subjected to a pipeline for genome assembly.

$k$-mer analysis was performed to survey genome size, heterozygosity and repeat content before genome assembly. The peak of $k$-mer frequency $(F)$ is determined by the total reads number $(N)$, genome size $(G)$, read length $(L)$ and the length of $k$-mer $(K)$, following the formula: $F=N \times(L-K+1) / G$. Total $k$-mer number $(M)$ is determined by the formula $M=N \times(L-K+1)$. As a result, genome size can be calculated by $G=M / F$. This formula enables accurate estimation of $G$, and hence an estimation of genome size for homozygous diploid or haploid genomes. All the above analyses indicated homozygosity of the genome and gave similar estimations of genome size. The final genome size estimate $(329 \mathrm{Mb}$ for $M$. viride and $85.68 \mathrm{Mb}$ for C. atmophyticus) was obtained through 17-mer analysis.

We carried out the SPAdes (v.3.10.1) $)^{55}$ genome assembly algorithm to assemble the contigs of $M$. viride. The contigs were formed without gaps. Subsequently platanus (v.1.2.4) was conducted to construct the scaffolds, from short insert-sized paired-end reads to long insert-sized paired-end reads. To extend the assembly and close gaps, the scaffolder SSPACE (v.3.0) ${ }^{56}$ was applied to extend the scaffolds while GapCloser (v.1.12) was used to close gaps and extend the scaffolds again. For C. atmophyticus, the assembly was generated by SOAPdenovo-127-mer (v.2.04) with a $k$-mer of 85 . To close the gaps within the constructed scaffolds, we used paired-end reads mapped to the scaffolds using GapCloser (v.1.12). Variant detection was done using Pilon (v.2.11) to improve draft assembly quality.

The quality of the assembly was evaluated in four ways. First, we used BUSCO $(v .3)^{58}$ to determine the proportion of a core-set of 303 highly conserved eukaryotic genes present in the genomes of M. viride and C. atmophyticus. Second, Soap (v.2.21) was used to map the reads to the draft assemblies to evaluate the DNA reads mapping rate in both species. Meanwhile, sequence depth and genetic copy content distribution were calculated. Third, we used BLAT (v.36) (v) $^{59}$ compare the draft assemblies to a transcript assembled by Bridger. Finally, we mapped the RNA reads to the draft assemblies to evaluate the RNA reads mapping rate using Tophat2 (ref. ${ }^{60}$ ).

Transcriptome sequencing and analysis. For Illumina sequencing, we considered two ways of library construction. The ribosomal RNA-depleted RNA library was constructed using the ribo-zero rRNA removal kit (plant) (Illumina) following the manufacturer's protocol, while the poly(A)-selected RNA library was constructed using the ScriptSeq Library Prep kit (Plant leaf) (Illumina) following the manufacturer's protocol. These libraries were sequenced on the Illumina sequencing system. All of the sequenced data were then assembled into transcripts following the Bridger pipeline. This set of transcript sequences was used for assessing the accuracy of the genome assembly and for gene annotation.

Gene expression was measured as fragments per kilobase of transcript per million mapped reads.

Detection and classification of repetitive elements. Three types of repeat (DNA transposon elements, retrotransposon elements and tandem repeats) were identified in the genomes of M. viride and C. atmophyticus. DNA transposons and retrotransposon elements were identified using MITE-hunter ${ }^{61}$ and LTRharvest ${ }^{62}$, 
respectively. RepeatModeler (v.1.0.8) was used to search for other repeats using a de novo approach. Alternatively, RepeatMasker ${ }^{63}$ was applied to using a custom library comprising a combination of Repbase and a de novo-predicted repetitive element library.

Gene prediction. We used a combination of de novo gene prediction methods, homology-based search methods and RNA sequencing-aided annotation methods. For de novo gene prediction, PASApipeline v.2.1.0 was applied to predict gene structure using transcripts assembled by Bridger, after which the inferred gene structures were used in AUGUSTUS (v.3.2.3) ${ }^{64}$ to train gene models based on transcript evidence. In addition, GeneMark (v.1.0) ${ }^{65}$ was used to build a hidden Markov model based on genome sequence. For homology-based annotation, we selected gene sets from certain model green algae. In regard to RNA sequencingaided methods, we took the transcripts assembled by Bridger as evidence. The final consensus gene sets were generated by combining all the evidence using MAKER (v.2.31.8) ${ }^{66}$. The result of the first round was used for SNAP to train another hidden Markov model based on transcriptome. Subsequently, the hidden Markov model was added to MAKER.

The final gene set was evaluated with two approaches. The BUSCO core eukaryotic gene-mapping approach was used to determine gene set completeness; RNA read mapping was another means of evaluation. We mapped the RNA reads to the gene set with Tophat 2 , while coverage depth was calculated by Samtools (v.0.1.19).

Gene function annotation was performed by BLASTP $\left(1 \times 10^{-5}\right)$ against several known databases, including SwissProt, TrEMBL, Kyoto Encyclopedia of Genes and Genomes (KEGG), COG and NR. InterProScan (using data from Pfam, PRINTS, SMART, ProDom and PROSITE) was used to identify protein motifs and protein domains of the predicted gene set. Gene Ontology information was obtained through Blast2go (v.2.5.0).

Comparative genome analyses and phylogenetics. The genomes of $M$. viride and C. atmophyticus were compared to those of nine other algae, namely Cyanophora paradoxa, Chondrus crispus, C. merolae, M. commoda, Ostreococcus tauri, Chlorella variabilis, Volvox carteri and Chlamydomonas reinhardtii, with the Streptophyta C. braunii, K. nitens, Physcomitrella patens, Selaginella moellendorffii, Oryza sativa subsp. Japonica and Arabidopsis thaliana. The same species were used to define orthogroups (using OrthoFinder, v.1.1.8). Single-copy gene families (that is, gene families with only one gene member per species) were used to construct phylogenetic trees based on maximum likelihood. We first performed multiple sequence alignment by MAFFT (v.7.310) for each single-copy gene orthogroup, followed by gap position removal (only positions where $50 \%$ or more of the sequences have a gap are treated as a gap position). A maximum-likelihood phylogenetic tree was constructed for each single-copy orthogroup. Next, we used ASTRAL to combine all single-copy gene trees to a species tree with the multispecies coalescent model. The online tool iTOL was performed to edit and display the final phylogenetic tree.

Gene identification. We used various search methods to identify different genes.

For TFs and TR we used the HMMER search method. We downloaded the HMMER model of the domain structure of each TF from the Pfam website (https://pfam.xfam.org/), referring to the TAPscan (v.2) TF database (https:// plantcode.online.uni-marburg.de/tapscan/). Preliminary candidates were collected by searching the HMM profile for each species $\left(<1 \times 10^{-10}\right)$. Then, we filtered genes that did not match the SwissProt functional annotation $\left(<1 \times 10^{-5}\right)$.

Finally, we filtered genes containing an incorrect domain according to the domain rules of the TAPscan (v.2) TF database. Most TFs/TRs were confirmed by phylogenetic analysis.

The HMMER search method was also used for phytohormone signalling pathways. We collected $\sim 10-20$ query genes from representative model organisms (for example, A. thaliana and C. reinhardtii). A custom profile HMM was built (hmmer-3.1b2) based on the query genes for each phytohormone. All signalling pathway genes must pass the following two restrictions: (1) genes should pass the HMM profile built by $\sim 10-20$ known genes $\left(<1 \times 10^{-10}\right)$, and (2) genes should match the SwissProt functional annotation $\left(<1 \times 10^{-5}\right)$. The custom profile HMM is available in the Supplementary Information (HMM profiles).

The genes involved in other pathways, such as biosynthetic phytohormone pathways and cell wall-related genes, were selected based on two criteria : (1) BLAST by known query genes $\left(<1 \times 10^{-5}\right)$, and (2) matching based on SwissProt functional annotation $\left(<1 \times 10^{-5}\right)$.

For CAZyme annotation we used the dbCAN2 metaserver (http://bcb.unl.edu/ dbCAN2/index.php). This server integrates three tools/databases for automated CAZyme annotation: (1) HMMER for annotation of the CAZyme domain against the dbCAN CAZyme domain HMM database; (2) DIAMOND for fast BLAST hits in the CAZy database; and (3) Hotpep for short conserved motifs in the PPR library.

We also constructed phylogenetic trees to classify certain highly similar genes, including ARF, CSL, HD-ZIP, YUCCA, PIF, TGA and others (see Supplementary Figs. 6-13). For phylogenetic analysis of individual genes, we first performed multiple sequence alignment by MAFFT (v.7.310) for each single-copy gene orthogroup, followed by removal of gap position (only positions where $50 \%$ or more of the sequences have a gap are treated as gap positions). Then, a maximumlikelihood phylogenetic tree was constructed by RAxML (amino acid substitution model: CAT + GTR, with 500 bootstrap replicates).

Analysis of significantly increased/decreased gene numbers of gene families. We used a median gene number to estimate the changes in gene family size ${ }^{6}$, shown in Fig. 1d. These showed the gene families whose numbers of genes were significantly increased in embryophytes compared to algae, by calculating the median of the embryophyte gene number/median of the algal gene number $\geq 10$. Taking Rhodophyta and Streptophyta as an example, we selected five representative species for each lineage. Gene numbers of these lineages in the gene family were sorted from largest to smallest. If the median of the Streptophyta gene number/ median of the Rhodophyta gene number was $>5$, this gene family was considered a significantly increased gene family in Streptophyta. The same method is used to define the embryophytes and algal genes in Fig. 1e. If the median gene number of both embryophytes and algae was $>0$, we defined the genes in this family as those commonly shared between embryophytes and algae. If the median embryophyte gene number was $>0$ and the median algae gene number was 0 , we defined the genes in this family as embryophyte genes. In special cases, if both the median embryophyte and median algae gene number was 0 , we removed those lowfrequency gene families.

Conserved motif identification. The local multiple Em (expectation maximization) for motif elicitation (MEME, http://meme-suite.org/) tool was used to identify conserved motifs. All genes in this study were analysed using the classical model. According to $e$ values, the number of motifs that MEME should find was set to 20 .

Reporting Summary. Further information on research design is available in the Nature Research Reporting Summary linked to this article.

\section{Data availability}

The whole-genome assemblies and transcriptome for M. viride and C. atmophyticus in this study are deposited at DDBJ/ENA/GenBank under accession nos. RHPH00000000 and RHPI00000000. Those data are also available in the CNGB Nucleotide Sequence Archive (accession no. CNP0000228).

Received: 4 March 2019; Accepted: 28 October 2019; Published online: 16 December 2019

\section{References}

1. Kenrick, P. \& Crane, P. The origin and early evolution of plants on land. Nature 389, 33-39 (1997).

2. Becker, B. \& Marin, B. Streptophyte algae and the origin of embryophytes. Ann. Bot. 103, 999-1004 (2009).

3. Delwiche, C. F. \& Cooper, E. D. The evolutionary origin of a terrestrial flora Curr. Biol. 25, R899-R910 (2015).

4. Delaux, P.-M. et al. Algal ancestor of land plants was preadapted for symbiosis. Proc. Natl Acad. Sci. USA 112, 13390-13395 (2015).

5. Bowman, J. L. et al. Insights into land plant evolution garnered from the Marchantia polymorpha genome. Cell 171, 287-304 (2017).

6. Hori, K. et al. Klebsormidium flaccidum genome reveals primary factors for plant terrestrial adaptation. Nat. Commun. 5, 3978 (2014).

7. Nishiyama, T. et al. The Chara genome: secondary complexity and implications for plant terrestrialization. Cell 174, 448-464 (2018).

8. Bowman, J. L., Briginshaw, L. N., Fisher, T. J. \& Flores-Sandoval, E. Something ancient and something neofunctionalized-evolution of land plant hormone signaling pathways. Curr. Opin. Plant Biol. 47, 64-72 (2019).

9. de Vries, J., Curtis, B. A., Gould, S. B. \& Archibald, J. M. Embryophyte stress signaling evolved in the algal progenitors of land plants. Proc. Natl Acad. Sci. USA 115, E3471-E3480 (2018).

10. de Vries, J., Stanton, A., Archibald, J. M. \& Gould, S. B. Streptophyte terrestrialization in light of plastid evolution. Trends Plant Sci. 21, 467-476 (2016).

11. Zhao, C. et al. Evolution of chloroplast retrograde signaling facilitates green plant adaptation to land. Proc. Natl Acad. Sci. USA 116, 5015-5020 (2019).

12. Mikkelsen, M. D. et al. Evidence for land plant cell wall biosynthetic mechanisms in charophyte green algae. Ann. Bot. 114, 1217-1236 (2014).

13. Wilhelmsson, P. K. I., Mühlich, C., Ullrich, K. K. \& Rensing, S. A. Comprehensive genome-wide classification reveals that many plant-specific transcription factors evolved in streptophyte algae. Genome Biol. Evol. 9, 3384-3397 (2017).

14. Lemieux, C., Otis, C. \& Turmel, M. A clade uniting the green algae Mesostigma viride and Chlorokybus atmophyticus represents the deepest branch of the streptophyta in chloroplast genome-based phylogenies. BMC Biol. 5, 2 (2007). 
15. Timme, R. E., Bachvaroff, T. R. \& Delwiche, C. F. Broad phylogenomic sampling and the sister lineage of land plants. PLoS ONE 7, e29696 (2012).

16. Wickett, N. J. et al. Phylotranscriptomic analysis of the origin and early diversification of land plants. Proc. Natl Acad. Sci. USA 111, E4859-E4868 (2014)

17. Wodniok, S. et al. Origin of land plants: do conjugating green algae hold the key? BMC Evol. Biol. 11, 104 (2011).

18. Cook, M. E. \& Graham, L. E. in Handbook of the Protists (eds Archibald, J. M. et al.) 1-20 (Springer International Publishing, 2016).

19. Ariel, F. D., Manavella, P. A., Dezar, C. A. \& Chan, R. L. The true story of the HD-Zip family. Trends Plant Sci. 12, 419-426 (2007).

20. Bouzroud, S. et al. Auxin response factors (ARFs) are potential mediators of auxin action in tomato response to biotic and abiotic stress (Solanum lycopersicum). PLoS ONE 13, e0193517 (2018).

21. Guan, H. et al. Genome-wide identification, phylogeny analysis, expression profiling, and determination of protein-protein interactions of the LEUNIG gene family members in tomato. Gene 679, 1-10 (2018).

22. Kaplan-Levy, R. N., Brewer, P. B., Quon, T. \& Smyth, D. R. The trihelix family of transcription factors - light, stress and development. Trends Plant Sci. 17, 163-171 (2012).

23. Mukherjee, K., Brocchieri, L. \& Bürglin, T. R. A comprehensive classification and evolutionary analysis of plant homeobox genes. Mol. Biol. Evol. 26, 2775-2794 (2009).

24. Omidbakhshfard, M. A., Proost, S., Fujikura, U. \& Mueller-Roeber, B. Growth-regulating factors (GRFs): a small transcription factor family with important functions in plant biology. Mol. Plant 8, 998-1010 (2015).

25. Paul, A. et al. RNA-seq-mediated transcriptome analysis of actively growing and winter dormant shoots identifies non-deciduous habit of evergreen tree tea during winters. Sci. Rep. 4, 5932 (2014).

26. Yoshida, T., Mogami, J. \& Yamaguchi-Shinozaki, K. ABA-dependent and ABA-independent signaling in response to osmotic stress in plants. Curr. Opin. Plant Biol. 21, 133-139 (2014).

27. Brenner, W. G., Romanov, G. A., Köllmer, I., Bürkle, L. \& Schmülling, T. Immediate-early and delayed cytokinin response genes of Arabidopsis thaliana identified by genome-wide expression profiling reveal novel cytokininsensitive processes and suggest cytokinin action through transcriptional cascades. Plant J. 44, 314-333 (2005)

28. Ulvskov, P., Paiva, D. S., Domozych, D. \& Harholt, J. Classification, naming and evolutionary history of glycosyltransferases from sequenced green and red algal genomes. PLoS ONE 8, e76511 (2013).

29. Petersen, B. L., Faber, K. \& Ulvskov, P. Glycosyltransferases of the GT77 family. Ann. Plant Rev. 41, 305-320 (2010).

30. Cheng, L. et al. Expressional characterization of galacturonosyltransferase-like gene family in Eucalyptus grandis implies a role in abiotic stress responses. Tree Genet. Genomes 14, 81 (2018).

31. Brawley, S. H. et al. Insights into the red algae and eukaryotic evolution from the genome of Porphyra umbilicalis (Bangiophyceae, Rhodophyta). Proc. Natl Acad. Sci. USA 114, E6361-E6370 (2017).

32. Minic, Z. \& Jouanin, L. Plant glycoside hydrolases involved in cell wall polysaccharide degradation. Plant Physiol. Biochem. 44, 435-449 (2006).

33. Tekle, Y. I., Wood, F. C., Katz, L. A., Cerón-Romero, M. A. \& Gorfu, L. A. Amoebozoans are secretly but ancestrally sexual: evidence for sex genes and potential novel crossover pathways in diverse groups of amoebae. Genome Biol. Evol. 9, 375-387 (2017).

34. Nevers, Y. et al. Insights into ciliary genes and evolution from multi-level phylogenetic profiling. Mol. Biol. Evol. 34, 2016-2034 (2017).

35. Keeling, P. J. \& Inagaki, Y. A class of eukaryotic GTPase with a punctate distribution suggesting multiple functional replacements of translation elongation factor 1. Proc. Natl Acad. Sci. USA 101, 15380-15385 (2004)

36. Cocquyt, E. et al. Gain and loss of elongation factor genes in green algae. BMC Evol. Biol. 9, 39 (2009).

37. Li, F. W. et al. Phytochrome diversity in green plants and the origin of canonical plant phytochromes. Nat. Commun. 6, 7852 (2015).

38. Romani, F., Reinheimer, R., Florent, S. N., Bowman, J. L. \& Moreno, J. E. Evolutionary history of HOMEODOMAIN LEUCINE ZIPPER transcription factors during plant transition to land. New Phytol. 219, 408-421 (2018).

39. Bari, R. \& Jones, J. D. G. Role of plant hormones in plant defence responses. Plant Mol. Biol. 69, 473-488 (2009).

40. Ju, C. et al. Conservation of ethylene as a plant hormone over 450 million years of evolution. Nat. Plants 1, 14004 (2015).

41. Van de Poel, B., Cooper, E. D., Van Der Straeten, D., Chang, C. \& Delwiche, C. F. Transcriptome profiling of the green alga Spirogyra pratensis (Charophyta) suggests an ancestral role for ethylene in cell wall metabolism, photosynthesis, and abiotic stress responses. Plant Physiol. 172, 533-545 (2016).

42. Han, G. Z. Evolution of jasmonate biosynthesis and signalling mechanisms. J. Exp. Bot. 68, 1323-1331 (2017)
43. Ohtaka, K., Hori, K., Kanno, Y., Seo, M. \& Ohta, H. Primitive auxin response without TIR1 and Aux/IAA in the charophyte alga Klebsormidium nitens. Plant Physiol. 174, 1621-1632 (2017).

44. Křeček, P. et al. The PIN-FORMED (PIN) protein family of auxin transporters. Genome Biol. 10, 249 (2009).

45. Viaene, T., Delwiche, C. F., Rensing, S. A. \& Friml, J. Origin and evolution of PIN auxin transporters in the green lineage. Trends Plant Sci. 18 , 5-10 (2013).

46. Yin, Y., Huang, J. \& Xu, Y. The cellulose synthase superfamily in fully sequenced plants and algae. BMC Plant Biol. 9, 99 (2009).

47. Simon, A., Glöckner, G., Felder, M., Melkonian, M. \& Becker, B. EST analysis of the scaly green flagellate Mesostigma viride (Streptophyta): implications for the evolution of green plants (Viridiplantae). BMC Plant Biol. 6, 1-13 (2006)

48. Del-Bem, L. E. Xyloglucan evolution and the terrestrialization of green plants. New Phytol. 219, 1150-1153 (2018).

49. Jensen, J. K. et al. Identification of an algal xylan synthase indicates that there is functional orthology between algal and plant cell wall biosynthesis. New Phytol. 218, 1049-1060 (2018).

50. Harholt, J., Moestrup, $\varnothing$. \& Ulvskov, P. Why plants were terrestrial from the beginning. Trends Plant Sci. 21, 96-101 (2016).

51. McFadden, G. I. \& Melkonian, M. Use of Hepes buffer for microalgal culture media and fixation for electron microscopy. Phycologia 25, 551-557 (1986).

52. Johnson, M. T. J. et al. Evaluating methods for isolating total RNA and predicting the success of sequencing phylogenetically diverse plant transcriptomes. PLoS ONE 7, e50226 (2012).

53. Rogers, S. O. \& Bendich, A. J. Extraction of DNA from milligram amounts of fresh, herbarium and mummified plant tissues. Plant Mol. Biol. 5, 69-76 (1985).

54. White Paper on De Novo Assembly in CLC Assembly Cell 4.0 (CLC bio A/S, 2012)

55. Bankevich, A. et al. SPAdes: a new genome assembly algorithm and its applications to single-cell sequencing. J. Comput. Biol. 19, 455-477 (2012).

56. Boetzer, M., Henkel, C. V., Jansen, H. J., Butler, D. \& Pirovano, W. Scaffolding pre-assembled contigs using SSPACE. Bioinformatics 27, 578-579 (2010)

57. Luo, R. et al. SOAPdenovo2: an empirically improved memory-efficient short-read de novo assembler. Gigascience 1, 18 (2012).

58. Simão, F. A., Waterhouse, R. M., Ioannidis, P., Kriventseva, E. V. \& Zdobnov, E. M. BUSCO: assessing genome assembly and annotation completeness with single-copy orthologs. Bioinformatics 31, 3210-3212 (2015).

59. Kent, W. J. BLAT-the BLAST-like alignment tool. Genome Res. 12, 656-664 (2002).

60. Kim, D. et al. TopHat2: accurate alignment of transcriptomes in the presence of insertions, deletions and gene fusions. Genome Biol. 14, R36 (2013).

61. Han, Y. \& Wessler, S. R. MITE-Hunter: a program for discovering miniature inverted-repeat transposable elements from genomic sequences. Nucleic Acids Res. 38, e199-e199 (2010).

62. Ellinghaus, D., Kurtz, S. \& Willhoeft, U. LTRharvest, an efficient and flexible software for de novo detection of LTR retrotransposons. BMC Bioinformatics 9, 18 (2008)

63. Chen, N. Using repeatmasker to identify repetitive elements in genomic sequences. Curr. Protoc. Bioinformatics 5, 4-10 (2004).

64. Stanke, M. et al. AUGUSTUS: ab initio prediction of alternative transcripts. Nucleic Acids Res. 34, W435-W439 (2006).

65. Besemer, J. \& Borodovsky, M. GeneMark: web software for gene finding in prokaryotes, eukaryotes and viruses. Nucleic Acids Res. 33, W451-W454 (2005).

66. Campbell, M. S. et al. MAKER-P: a tool kit for the rapid creation, management, and quality control of plant genome annotations. Plant Physiol. 164, 513-524 (2014)

\section{Acknowledgements}

We thank G. Günther (http://www.mikroskopia.de/index.html), who took microscopic images of $M$. viride and C. atmophyticus. Financial support was provided by the Shenzhen Municipal Government of China (grant nos. JCYJ20151015162041454 and JCYJ20160531194327655) and the Guangdong Provincial Key Laboratory of Genome Read and Write (grant no. 2017B030301011). This work is part of the 10KP project led by BGI-Shenzhen and China National GeneBank.

\section{Author contributions}

X.L., M.M. and H. Liu conceived, designed and supervised the project. X.L., M.M., B.M., H. Liu., X.X., J.W., H.Y., Y.V.P. and G.K.-S.W. provided resources and materials. Z.C. and S.K.S. developed the protocol for DNA extraction. S. Wittek and T.R. grew the organisms to quantity and extracted DNA. Samples were sequenced by BGI. S.Wang. and L.L. generated the draft genome and performed the annotation. S.Wang., L.L., H. Li., S.K.S., H.W., Y.X., W.X., B.S., H. Liang, S.C., Y.C., Y.S. and M.P. analysed data. S. Wang., L.L., S.K.S. and M.M. wrote the paper. 


\section{Competing interests}

The authors declare no competing interests.

\section{Additional information}

Extended data is available for this paper at https://doi.org/10.1038/s41477-019-0560-3. Supplementary information is available for this paper at https://doi.org/10.1038/ s41477-019-0560-3.

Correspondence and requests for materials should be addressed to G.K.-S.W., M.M., H.L. or X.L.

Peer review information Nature Plants thanks John Bowman, Stefan Rensing, Charles Wellman and the other, anonymous, reviewer(s) for their contribution to the peer review of this work.

Reprints and permissions information is available at www.nature.com/reprints.
Publisher's note Springer Nature remains neutral with regard to jurisdictional claims in published maps and institutional affiliations.

Open Access This article is licensed under a Creative Commons

Attribution 4.0 International License, which permits use, sharing, adaptation, distribution and reproduction in any medium or format, as long as you give appropriate credit to the original author(s) and the source, provide a link to the Creative Commons license, and indicate if changes were made. The images or other third party material in this article are included in the article's Creative Commons license, unless indicated otherwise in a credit line to the material. If material is not included in the article's Creative Commons license and your intended use is not permitted by statutory regulation or exceeds the permitted use, you will need to obtain permission directly from the copyright holder. To view a copy of this license, visit http://creativecommons. org/licenses/by/4.0/.

(c) The Author(s) 2019 
a

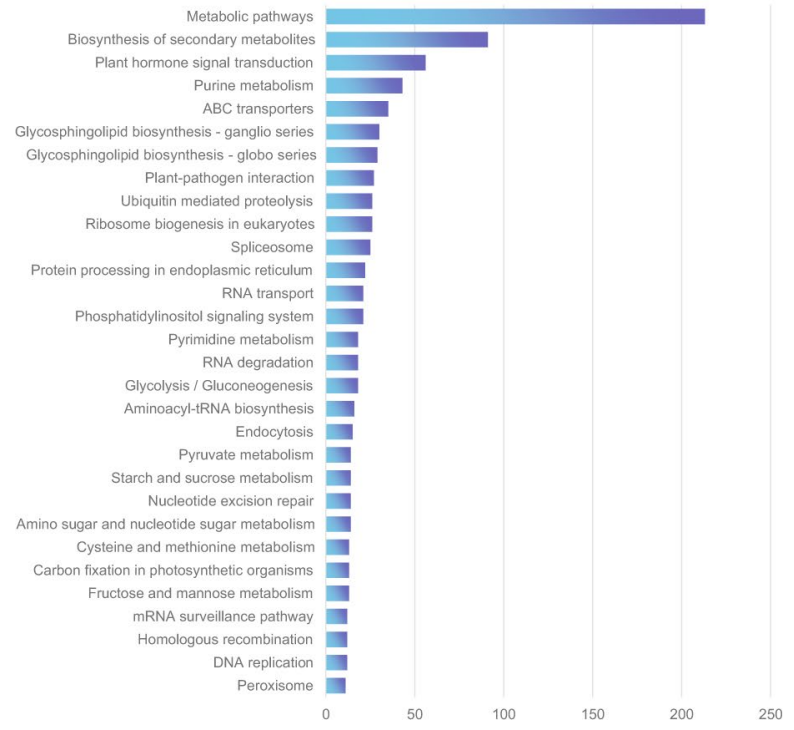

b

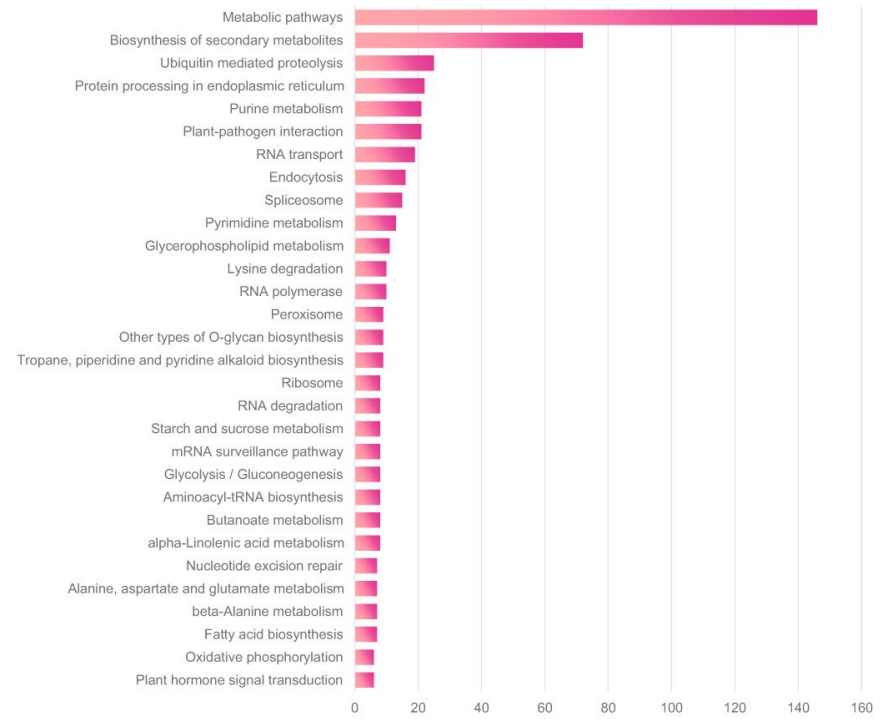

Extended Data Fig. 1 | The KEGG distribution of unique proteins in M. viride (blue) and C. atmophyticus (pink). The x-axis indicates the number of genes in a specific category in the respective species. The metabolism pathway is shown on the $y$-axis. 


\begin{tabular}{|c|c|c|c|c|c|c|c|}
\hline GTG & AHK & BAK1 & $\mathrm{CKI}$ & ERS & ETR & PIN & \\
\hline 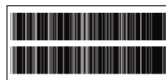 & 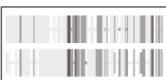 & 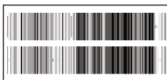 & 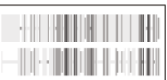 & 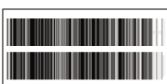 & 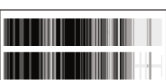 & $\mid$ & O. sativa \\
\hline 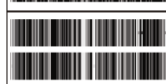 & 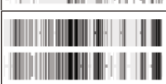 & 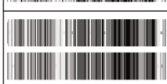 & $\begin{array}{l}\|\|\|\| \| \\
\|\|\|\| \|\end{array}$ & 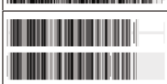 & 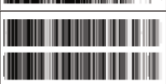 & 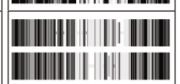 & S. moellendorffii \\
\hline & | & 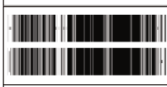 & 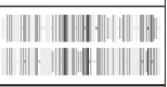 & 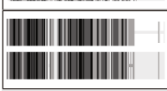 & 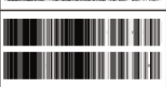 & 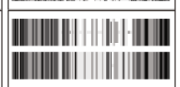 & P. patens \\
\hline 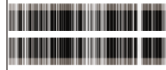 & inin & 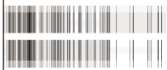 & 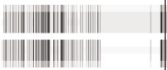 & 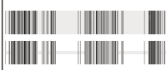 & 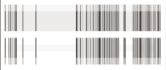 & 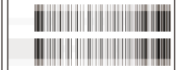 & C. braunii \\
\hline 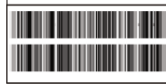 & 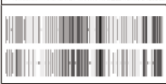 & 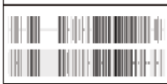 & 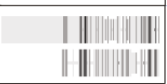 & 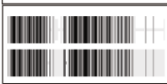 & 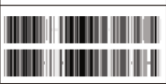 & 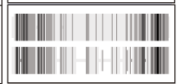 & K. nitens \\
\hline 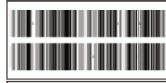 & 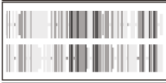 & 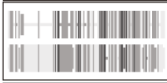 & 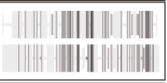 & 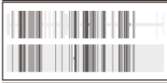 & 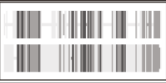 & & C. atmophyticus \\
\hline 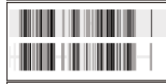 & 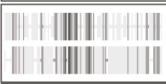 & 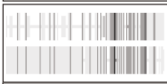 & 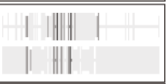 & 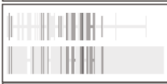 & 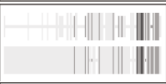 & 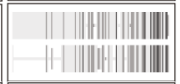 & M. viride \\
\hline 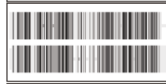 & $\begin{array}{l}\|\|\|H\| \| \\
\|\|\|\| \|\end{array}$ & \begin{tabular}{|l|||||||||||||||||}
|||||||||||||| $\mid$ \\
\end{tabular} & 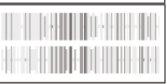 & \begin{tabular}{c|}
$\||||||||| \mid$ \\
$\|||||||||||$ \\
\end{tabular} & $\begin{array}{l}\|\|\|\| \| \\
\|\|\|\mid\| \\
\end{array}$ & & V. carteri \\
\hline 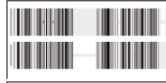 & $\begin{array}{l}\|\| \| \\
\|\| \| \\
\end{array}$ & 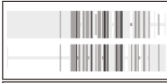 & 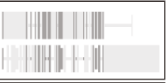 & 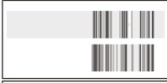 & $\begin{array}{l}\|\|\|\| \| \\
\|\|\|\|\end{array}$ & & C. reinhardtii \\
\hline $\begin{array}{l}\||||||||||||||||||| \\
|\|||||||||||||||| \mid\end{array}$ & $\begin{array}{l}\|\|\|\| \\
\|\| \|\end{array}$ & 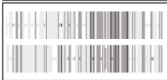 & \| \|\|\|\|\|\|\|\|$\|$ & 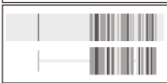 & ||\||||||| & & M. pusilla \\
\hline & & 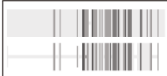 & & & & & P. umbilicalis \\
\hline & & 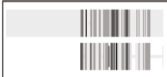 & & & & & C. crispus \\
\hline & & 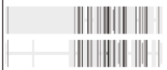 & & & & & C. merolae \\
\hline
\end{tabular}

Extended Data Fig. 2 | Sequence conservation of various phytohormone receptor genes in representative species. Each cell shows a pairwise sequence alignment between a known Arabidopsis protein receptor (top) and the best BLAST hit (E-value $<1 e-10$ ) in the translated genome of the indicated species. Black and grey represent similar amino acids (the darker the bars, the higher is the similarity). 


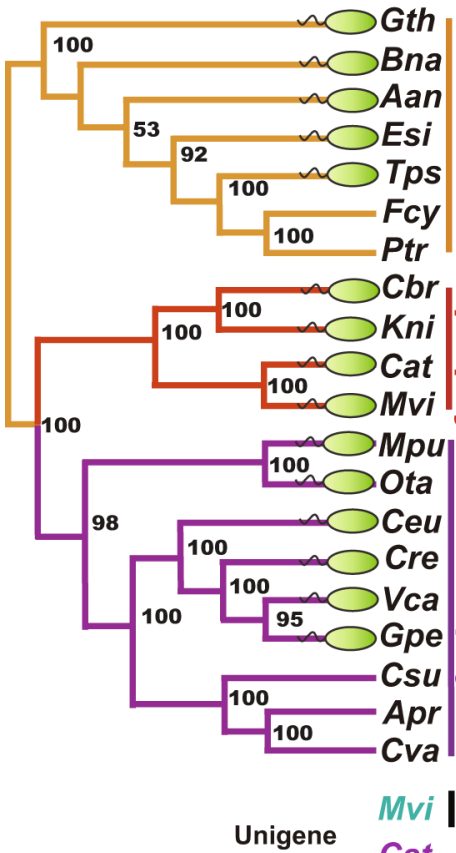

Unigene Mvi
Central Pair Radial Spoke Protein Protein Out dynein dynein
Inner Intraflagellar Transport

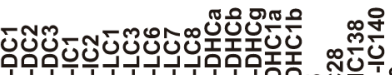
Protein
Dynein protien

우두눈꾼

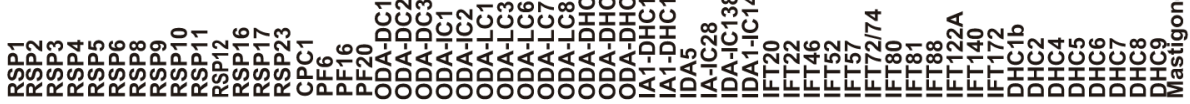

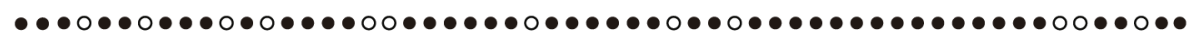

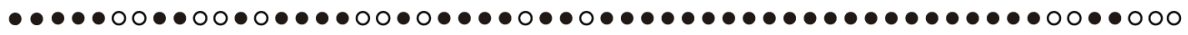

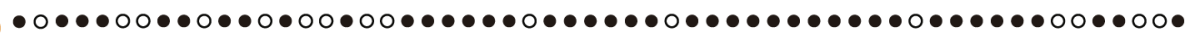

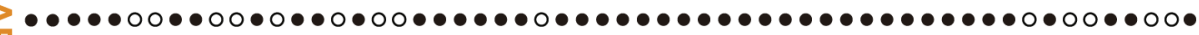

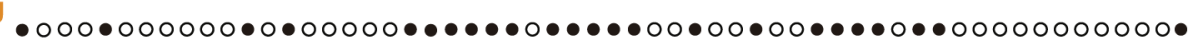
$\bullet 0000000000 \bullet 0 \bullet 0000000 \bullet \bullet 0 \bullet 00 \bullet 00000 \bullet 000000000000000 \bullet 00000000$ S $0000000000 \bullet 0 \bullet 0000000 \bullet 0000 \bullet \bullet 00000 \bullet 00 \bullet 000000000000 \bullet 00000000$

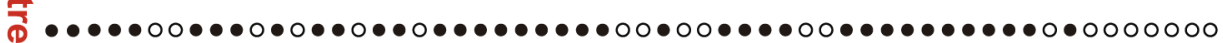

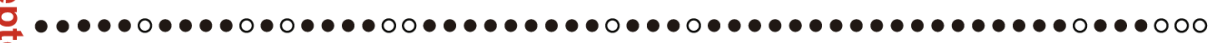

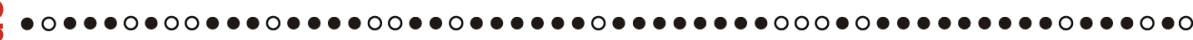

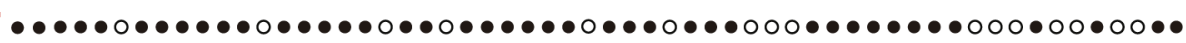

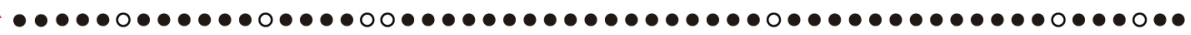
-

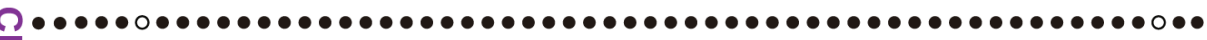
כ $\bullet \bullet \bullet \bullet \bullet \bullet \bullet \bullet \bullet \bullet \bullet \bullet \bullet \bullet \bullet \bullet \bullet \bullet \bullet \bullet \bullet \bullet \bullet \bullet \bullet \bullet \bullet \bullet \bullet \bullet \bullet \bullet \bullet \bullet \bullet \bullet \bullet \bullet \bullet \bullet \bullet \bullet \bullet \bullet \bullet \bullet \bullet \bullet \bullet \bullet \bullet \bullet \bullet \bullet \bullet \circ \bullet$

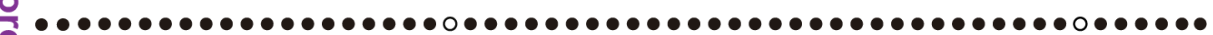

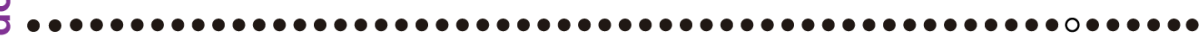
$\bullet$

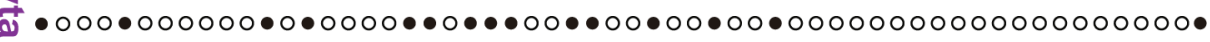

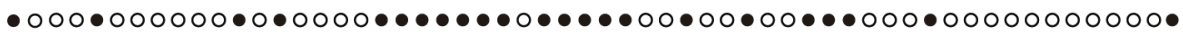

Transcript Level

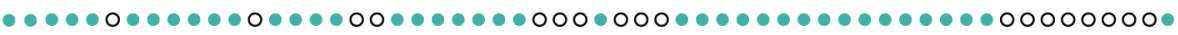

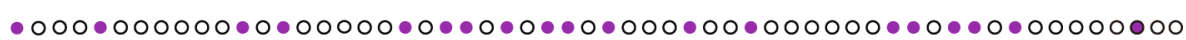
$100 \%$ $0 \%$ 旦 $\square$ C.atmophyticus $\square$ M. viride

Extended Data Fig. 3 | Analysis of the conserved flagellar proteome in flagellate and non-flagellate organisms and the distribution of key flagellar proteins. Key structure-related flagellar proteins in flagellate and non-flagellate algal species in different lineages. The phylogenetic tree on the left panel was constructed using maximum-likelihood method based on the concatenated sequences of single-copy genes from these genomes, after excluding the species-specific gene duplications. The presence (filled circle) or absence (empty circle) of putative orthologs to conserved flagellar proteins is shown on right panel [Based on Reciprocal Blast Hit (RBH) method with Cut-off value of e-5]. The histogram on the lower panel shows the differential expression level of these important structure-related flagellar proteins. 


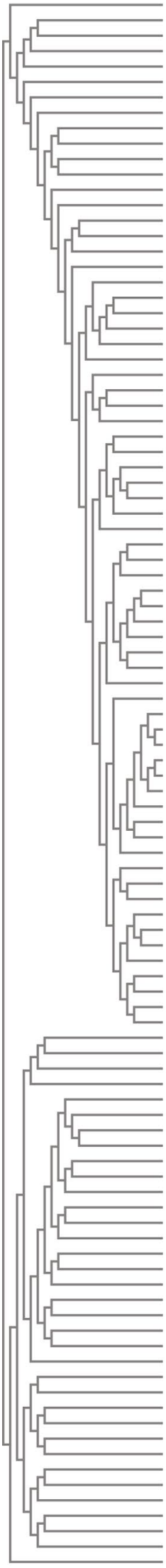

Colsc7335S0T7361-Coleochaete_scutata-S_algae

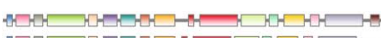

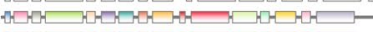
OSX74984.1-Red_algae

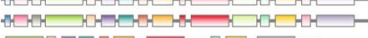
OSX75725.1-Red algae CMER CMH226C Red algae Cyapa7815C30256-G algae Cocsu1898-Coccomyxa subellipsoidea-C_algae Mesvi514S00657-Mesostigma-S_algae Spisp1220S22468-Spirotaenia endospira-S algae Spisp164S08392-Spirotaenia_endospira-S àlgae Spisp50S23044-Spirotaenia endospira-S algae Spisp343S13491-Spirotaenia endospira-S algae Chrsp80S07794-Chlorokybus-S algae KleflGAQ80114.1-Klebsormidium nitens-S algae Mesen70S09236-Mesotaenium en endlicherianum-S alga Colsc25891S0T22366-Coleochaete_scutata-S_algae

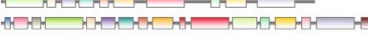

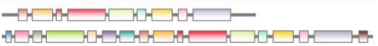

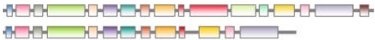

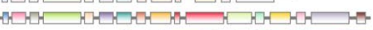

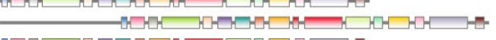

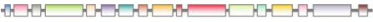

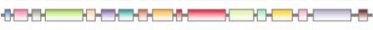
th M픈 Colsc32S0T70-Coleochaete_scutatā-S algae Colsc23S0T66-Coleochaete_scutata-S_algae g5750-Chara braunii-S algae

g19907-Charā braunii-S algae

19905-Chara braunii-S algae

g19903-Chara-brauni-S algae g41262-Chara braunii-S algae 11889-Chara braunii-S alga Mapoly0038s0022.1.p Marchantia polymorpha Mapoly0145s0014.1. - Marchantia polymoroha Mapoly0145s0017.1.p Marchantia polymorpha Mapoly0145s0015.1. Marchantia polymorpha Mapoly0002s0240.1. Marchantia polymorpha Mapoly0022s0170.1. Marchantia polymorpha Mapoly0024s0116 1. Marchantia polymorpha Mapoly0038s00231. Marchantia polymorpha Mapoly0145s016.1. Marchantia_polymorpha Mapoly052s0109.1. Marchantia_polymorpha Mapoly0104s0043.1. Marchantia polymorpha Azfi s0291 063341-Ázolla filiculoides-FERN Azfi - 0004 g008556-Azolla-filiculoides-FERN Azfi s0004.g008493-Azolla filiculoides-FERN Sacu_v1.1_s0046.g013360-Salvinia_cucullata-FERN Sacu- v1.1 ${ }^{-}$s0021.g008259-Salvinia_cucullata-FERN Sacu_v1.1_s0103.g019963-Salvinia_cucullata-FERN Sacu_v1.1_s0028.g009882-Salvinia_cucullata-FERN Sacu-v1.1-s0036.g011665-Salvinia_cucullata-FERN Sacu- v1. $1^{-}$s0015.g006537-Salvinia cucullata-FERN Azfi_s0002.g001607-Azolla filiculoides-FERN Pp1s7 451V6.4-Physcomitrella patens-MOSS Pp1s84 186V6.1-Physcomitrella patens-MOSS Pp1s59-188V6.3-Physcomitrella_patens-MOSS Pp1s59-181V6.1-Physcomitrella_patens--MOSS Pp1s59-167V6. 3-Physcomitrella-patens-MOSS Pp1s59-161V6.1-Physcomitrella patens-MOSS Pp1s59-163V6.1-Physcomitrella_patens-MOSS Pp1s7 $394 V 6$. 1-Physcomitrella patens-MOSS Pp1s7 757 V6.1-Physcomitrella patens-MOSS Pp1s7 ${ }^{-}$445V6.3-Physcomitrella_patens-MOSS P115263 8V6. 1-Physcomitrella patens-MOSS PACid 15421849 Selaginella moellendorffii PACid 15404946 Selaginella-moellendorffi PACid 15409631 - Selaginella - moellendorffii Os $03+0178000-01$-Oryza sativa-land palnt Os03t0177500-01-Oryza_sativa-land_palnt os03t0177400-01-Oryza_sativa-land palnt Os03t0177900-01-Oryza sativa-land palnt AT1G07940 2-Arabidopsis thaliana-land palnt AT1G07930.1-Arabidopsis thaliana-land paln AT5G60390 3-Arabidopsis thaliana-land paln AT1G07920.1-Arabidopsis thaliana-land paln Colsc14338S0T20518-Coleochaete_scutata-S_algae Mesvi72S00837-MesostigmaS_algae ABD58901.1-Mesostigma_viride

COLL63 NEPOL-Nephroselmis_olivacea

Volca350-Volvox_carteri-C_algae

Volca2340-Volvox_carteri-C_algae

Gonpe4831-Gonium_pectorale-C_algae

Gonpe 13750-Gonium_pectorale-C_algae

Chlre12766-Chlamydomonas_reinhardtii-C_algae

Chlre1331-Chlamydomonas reinhardtii-C_algae

COLL59 CHLRE-Chlamydomonas reinhar

Chlva3627-Chlorella_variabilis-C_algae

Chlva3624-Chlorella variabilis-C ${ }^{-}$algae
Micco3447-Micractinium conductrix-C algae

Chrzo02g32220.t1-Chromochloris_zofingiensis-C_algae

Chrzo16g05140.t1-Chromochloris zofingiensis-C- algae

COLL60 TETOB-Tetradesmus obliquus

COLL58-ULVIN-Ulva intestinalis

COLL57-9CHLO-Ulvā fenestrata

COLL55 9CHLO-Ulvella repens

COLL62-TETST-TetraseImis striata

Batpr2190-Bathycoccus_prasinos-C algae K8F4B8 9CHLO-Bathycoccus prasinos MicRCC 4377 -Micromonas commoda-C algae MicRCC4580-Micromonas commoda-C ${ }^{-}$algae Micpu3913-Micromonas_pūsilla-C_algae Micpu6175-Micromonas pusilla-C algae Ostlu7504-Ostreococcus lucimarinus-C algae Ostlu5598-Ostreococcus lucimarinus- $C$-algae Ostlu5732-Ostreococcus lucimarinus-C algae Ostta5975-Ostreococcus tauri-C_algae Ostta5831-Ostreococcus tauri-C algae COLL64 OSTTA-Ostreococcus taurí CDF32437.1-Red algae

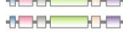

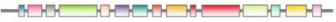

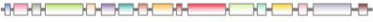

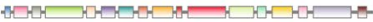

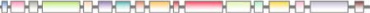

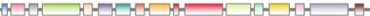

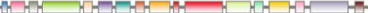

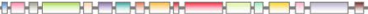

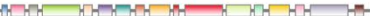

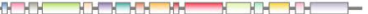

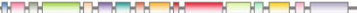

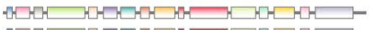

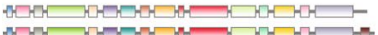

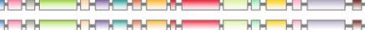

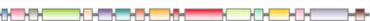

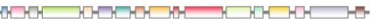

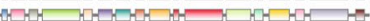

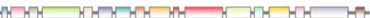

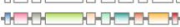

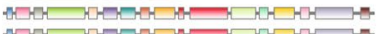

스만든

NHer

An

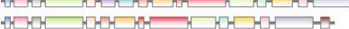

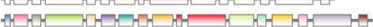

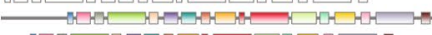

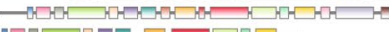

tous

the

15

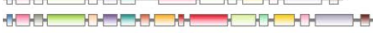

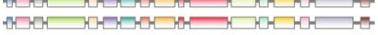

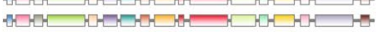

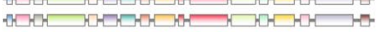

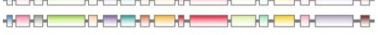

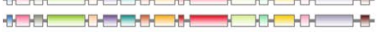

4-

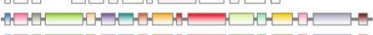

반다난

느는

40

40

trat

wen

at

tra

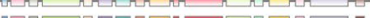

t-

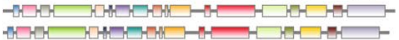

प्रा

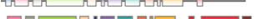

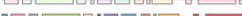

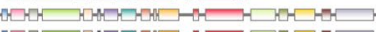

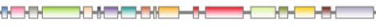

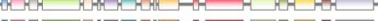

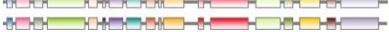

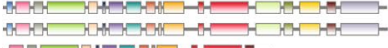

10

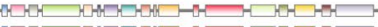

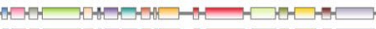

we the ant

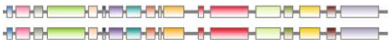

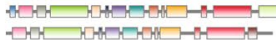

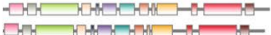

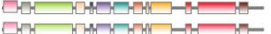

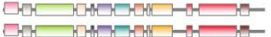

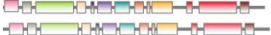

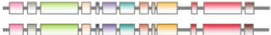

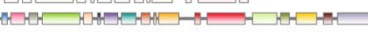

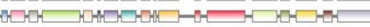

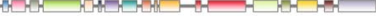

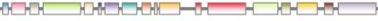

HeFr CHA

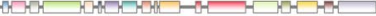

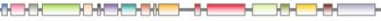

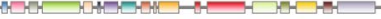

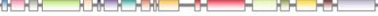

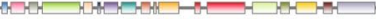

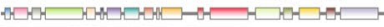

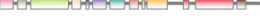

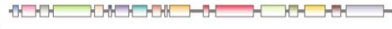

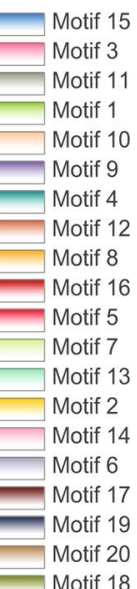

Motif 18

$\begin{array}{llllllll}0.0 & 100.0 & 200.0 & 300.0 & 400.0 & 500.0 & 600.0 & 700.0\end{array}$

Extended Data Fig. 4 | Distribution of EF-1 $\alpha$ and EF-like motifs. Maximum Likelihood was used to infer the phylogenetic tree of the EF-1 $\alpha$ and EF-like homologs to understand their phylogenetic distribution. The right panel displays the representative EF-1 $\alpha$ and EF-like motifs from red algae to embryophytes. The tree derived from a MAFFT alignment and constructed using IQ-TREE (see Methods). Bootstrap values (500 replicates) $\geq 50 \%$ are shown. 


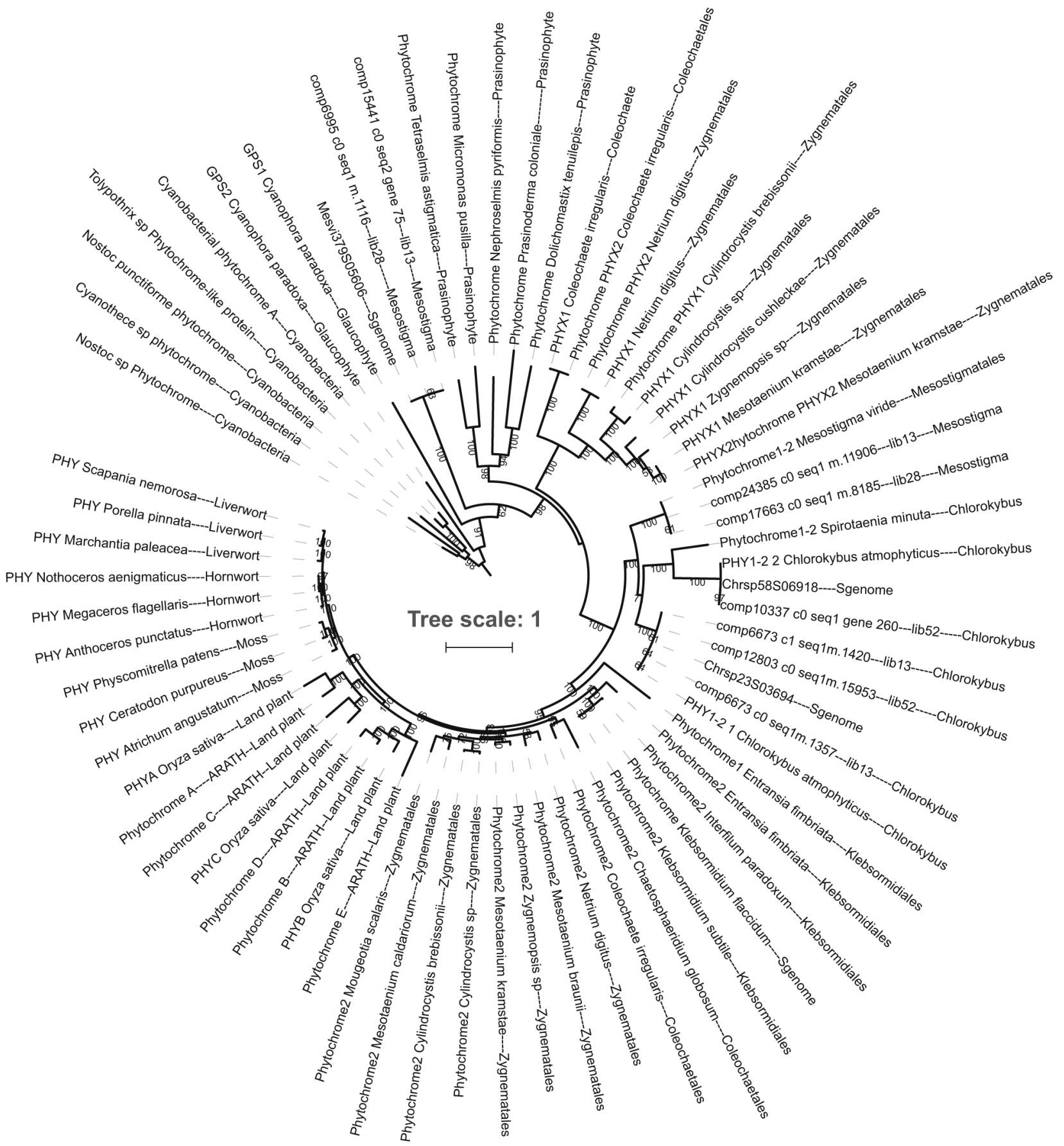

Extended Data Fig. 5 | Phylogenetic tree of phytochrome. Maximum Likelihood was used to infer the phylogenetic tree of the phytochrome. The tree derived from a MAFFT alignment and constructed using RAxML (see Methods). Bootstrap values (500 replicates) $\geq 50 \%$ are shown. 


\section{Reporting Summary}

Nature Research wishes to improve the reproducibility of the work that we publish. This form provides structure for consistency and transparency in reporting. For further information on Nature Research policies, see Authors \& Referees and the Editorial Policy Checklist.

\section{Statistics}

For all statistical analyses, confirm that the following items are present in the figure legend, table legend, main text, or Methods section.

n/a Confirmed

Х $\square$ The exact sample size $(n)$ for each experimental group/condition, given as a discrete number and unit of measurement

Х $\square$ A statement on whether measurements were taken from distinct samples or whether the same sample was measured repeatedly

$\triangle \square$ The statistical test(s) used AND whether they are one- or two-sided

X Only common tests should be described solely by name; describe more complex techniques in the Methods section.

$\triangle \square$ A description of all covariates tested

\ $\square$ A description of any assumptions or corrections, such as tests of normality and adjustment for multiple comparisons

A full description of the statistical parameters including central tendency (e.g. means) or other basic estimates (e.g. regression coefficient)

AND variation (e.g. standard deviation) or associated estimates of uncertainty (e.g. confidence intervals)

$\square$ For null hypothesis testing, the test statistic (e.g. $F, t, r$ ) with confidence intervals, effect sizes, degrees of freedom and $P$ value noted Give $P$ values as exact values whenever suitable.

Х $\square$ For Bayesian analysis, information on the choice of priors and Markov chain Monte Carlo settings

Х $\square$ For hierarchical and complex designs, identification of the appropriate level for tests and full reporting of outcomes

$\triangle \square$ Estimates of effect sizes (e.g. Cohen's $d$, Pearson's $r$ ), indicating how they were calculated

\section{Our web collection on statistics for biologists contains articles on many of the points above.}

\section{Software and code}

Policy information about availability of computer code

Data collection

Paired-end libraries with insert sizes of $170 \mathrm{bp}, 250 \mathrm{bp}, 500 \mathrm{bp}, 800 \mathrm{bp}, 2 \mathrm{~kb}, 5 \mathrm{~kb}, 10 \mathrm{~kb}$ and $20 \mathrm{~kb}$ were constructed following standard Illumina protocols. The libraries were sequenced on an Illumina HiSeq 2000/4000 and BGI-seq 500 platform. A total of 245Gb (about 746.86X) and 66.46Gb (about 775.68X) paired-end data were generated for M. viride (CCAC 1140) and C. atmophyticus (CCAC 0220), respectively.

For Illumina sequencing, we considered two ways of library construction. The rRNA-depleted RNA library was constructed using the ribozero rRNA removal kit (plant) (Illumina, American) following the manufacturer's protocol, while the poly (A)-selected RNA library was constructed using the ScriptSeq Library Prep kit (Plant leaf) (Illumina, American) following the manufacturer's protocol.

Data analysis

The list of Software used in this study are as follows:

CLC Assembly Cell (version 5.0.1)

Pairfa (version 0.16.0)

SOAPfilter (version 2.2)

Kmerfreq (version 1.0)

SPAdes (version 3.10.1)

Platanus (version 1.2.4)

SOAPdenovo-127mer (version 2.04)

SSPACE (version 3.0)

GapCloser (version 1.12)

Pilon (version 2.11)

BUSCO (version3)

Soap (version 2.21)

blat (v36)

Bridger_r2014-12-01 
Trinityrnaseq (version 2.1.1)

Tophat2 (version 2.1.0)

RepeatModeler (version 1.0.8)

GenomeTools (version 1.5.8)

MITE-hunter (version 8/19/2010)

LTRharvest (version 1.0)

PASApipeline-2.1.0

AUGUSTUS (version 3.2.3)

GeneMark (version 1.0)

MAKER (version 2.31.8)

SNAP (version 2006-07-28)

Samtools (version 0.1.19)

blast-2.2.26

ncbi-blast-2.2.31+

Blast2go (version 2.5.0).

InterProScan 5.28-67.0

OrthoFinder (version 1.1.8)

hmmer-3.1b2

MAFFT (version 7.310)

RAXML (version 8.2.4)

IQ-tree (version 1.6.1)

ASTRAL (version 4.11.1)

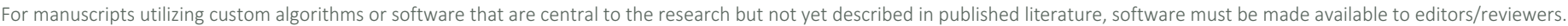
We strongly encourage code deposition in a community repository (e.g. GitHub). See the Nature Research guidelines for submitting code \& software for further information.

\section{Data}

Policy information about availability of data

All manuscripts must include a data availability statement. This statement should provide the following information, where applicable:

- Accession codes, unique identifiers, or web links for publicly available datasets

- A list of figures that have associated raw data

- A description of any restrictions on data availability

The whole genome assemblies for M. viride and C. atmophyticus in this study are deposited at DDBJ/ENA/GenBank under the accession numbers of RHPH00000000 and RHPI00000000. Those data are also available in the CNGB Nucleotide Sequence Archive (CNSA: http://db.cngb.org/cnsa; accession number CNA0002352 and CNA0002353).

\section{Field-specific reporting}

Please select the one below that is the best fit for your research. If you are not sure, read the appropriate sections before making your selection.

\Life sciences

Behavioural \& social sciences

Ecological, evolutionary \& environmental sciences

For a reference copy of the document with all sections, see nature.com/documents/nr-reporting-summary-flat.pdf

\section{Life sciences study design}

All studies must disclose on these points even when the disclosure is negative.

Sample size Axenic cultures of Mesostigma viride (CCAC 1140) and Chlorokybus atmophyticus (CCAC 0220) were obtained from the Central Collection of Algal Cultures (CCAC; http://www.ccac.uni-koeln.de/) and grown in Waris-H culture medium (McFadden and Melkonian 1986). No statistical methods were used to predetermine sample sizes.

Data exclusions

The reads with low quality are more likely to contain errors, which might complicate the assembly process, and were thus excluded. To reduce the effect of sequencing error on assembly, we performed the quality control of raw data using the default parameters. Briefly, We used CLC Assembly Cell (version 5.0.1) to trim the adapters, remove duplicates and trim low quality bases. Then, we used Pairfq (version 0.16.0) (https://github.com/sestaton/Pairfq) to pair the reads. Finally, SOAPfilter (version 2.2) was used to filter the reads again. After filtering off duplicated reads, low quality reads and reads with adaptor sequences, $74.63 \mathrm{~Gb}$ and $14.51 \mathrm{~Gb}$ high-quality clean reads remained for $\mathrm{M}$. viride (CCAC1140) and C. atmophyticus (CCAC 0220), respectively, which were then subjected to a pipeline for genome assembly.

Replication Since it was a Genome sequencing project, without any experiments. All the data were generated from a single axenic culture. Therefore, no replications were required.

Randomization Since it was a Genome sequencing project, without any experiments. All the data were generated from a single axenic culture. Therefore, no randomizations were required.

Blinding

Since it was a Genome sequencing project, without any experiments. All the data were generated from a single axenic culture. Therefore, no blinding experiments were required. 


\section{Reporting for specific materials, systems and methods}

We require information from authors about some types of materials, experimental systems and methods used in many studies. Here, indicate whether each material, system or method listed is relevant to your study. If you are not sure if a list item applies to your research, read the appropriate section before selecting a response.

\begin{tabular}{|c|c|c|c|}
\hline \multicolumn{2}{|r|}{ Materials \& experimental systems } & \multicolumn{2}{|c|}{ Methods } \\
\hline $\mathrm{n} / \mathrm{a}$ & Involved in the study & $\mathrm{n} / \mathrm{a}$ & Involved in the study \\
\hline Х & Antibodies & Х & $\square$ ChIP-seq \\
\hline Х & Eukaryotic cell lines & Х & $\square$ Flow cytometry \\
\hline$\bigotimes$ & Palaeontology & Х & MRI-based neuroimaging \\
\hline$\bigotimes$ & $\square$ Animals and other organisms & & \\
\hline$\bigotimes$ & $\square$ Human research participants & & \\
\hline$\bigotimes$ & $\square$ Clinical data & & \\
\hline
\end{tabular}

